\title{
Article
}

\section{Low-Cost Calibration of Matching Error between Lidar and Motor for a Rotating 2D Lidar}

\author{
Chang Yuan ${ }^{1}$, Shusheng Bi ${ }^{1, *}$, Jun Cheng ${ }^{1}$, Dongsheng Yang ${ }^{2}\left(\mathbb{C}\right.$ and Wei Wang ${ }^{3}$ \\ 1 Robotics Institute, Beihang University, Beijing 100191, China; yuanchang@buaa.edu.cn (C.Y.); \\ chengjun@buaa.edu.cn (J.C.) \\ 2 Tencent, Beijing 100094, China; eastyang@tencent.com \\ 3 Institute of Informatization and Industrialization Integration, China Academy of Information and \\ Communications Technology (CAICT), Beijing 100191, China; wangwei15@caict.ac.cn \\ * Correspondence: ssbi@buaa.edu.cn
}

Citation: Yuan, C.; Bi, S.; Cheng, J.;

Yang, D.; Wang, W. Low-Cost

Calibration of Matching Error between Lidar and Motor for a Rotating 2D Lidar. Appl. Sci. 2021, 11, 913. https://doi.org/10.3390/ app11030913

Received: 15 November 2020 Accepted: 4 January 2021 Published: 20 January 2021

Publisher's Note: MDPI stays neutral with regard to jurisdictional clai$\mathrm{ms}$ in published maps and institutional affiliations.

Copyright: (C) 2021 by the authors. Licensee MDPI, Basel, Switzerland. This article is an open access article distributed under the terms and conditions of the Creative Commons Attribution (CC BY) license (https:// creativecommons.org/licenses/by/ $4.0 /)$.

\begin{abstract}
For a rotating 2D lidar, the inaccurate matching between the 2D lidar and the motor is an important error resource of the 3D point cloud, where the error is shown both in shape and attitude. Existing methods need to measure the angle position of the motor shaft in real time to synchronize the 2D lidar data and the motor shaft angle. However, the sensor used for measurement is usually expensive, which can increase the cost. Therefore, we propose a low-cost method to calibrate the matching error between the 2D lidar and the motor, without using an angular sensor. First, the sequence between the motor and the $2 \mathrm{D}$ lidar is optimized to eliminate the shape error of the $3 \mathrm{D}$ point cloud. Next, we eliminate the attitude error with uncertainty of the 3D point cloud by installing a triangular plate on the prototype. Finally, the Levenberg-Marquardt method is used to calibrate the installation error of the triangular plate. Experiments verified that the accuracy of our method can meet the requirements of the 3D mapping of indoor autonomous mobile robots. While we use a 2D lidar Hokuyo UST-10LX with an accuracy of $\pm 40 \mathrm{~mm}$ in our prototype, we can limit the mapping error within $\pm 50 \mathrm{~mm}$ when the distance is no more than $2.2996 \mathrm{~m}$ for a $1 \mathrm{~s}$ scan (mode 1 ), and we can limit the mapping error within $\pm 50 \mathrm{~mm}$ at the measuring range $10 \mathrm{~m}$ for a $16 \mathrm{~s}$ scan (mode 7). Our method can reduce the cost while the accuracy is ensured, which can make a rotating 2D lidar cheaper.
\end{abstract}

Keywords: 2D lidar; rotating 2D lidar; calibration; low cost

\section{Introduction}

As an environmental modeling sensor, lidar is widely used. Typically, lidar can be divided into 2D lidar and 3D lidar, 3D lidar can scan 3D surfaces and obtain 3D maps of surroundings, but it is usually quite expensive. While the price of 2D lidar is relatively cheap, it can only obtain 2D maps which contain less information than 3D maps. However, if a 2D lidar is moved in a certain direction, it can be used to scan a 3D surface [1]. By moving a 2D lidar, one can model a 3D environment at low cost. Thus, a moving 2D lidar can replace (or at least partially replace) a commercial 3D lidar in many applications, avoiding the expensive cost of using a 3D lidar.

To build a moving 2D lidar, a common way is to install a 2D lidar on a motor shaft, so that a rotating $2 \mathrm{D}$ lidar is built $[2,3]$. The data collected by a $2 \mathrm{D}$ lidar is combined with the rotation angle of a motor, and the 3D coordinates of the points are calculated. Research on rotating 2D lidars can be divided into two categories, one is how to eliminate the error of the $3 \mathrm{D}$ point cloud while a rotating $2 \mathrm{D}$ lidar is working in a static environment [1-23], the other is how to correct the distortion of the 3D point cloud while a rotating 2D lidar is working in dynamic environment [24-28]. In this paper, we focus on the former question, that is, how to eliminate the error of a rotating $2 \mathrm{D}$ lidar while it is working in a static environment. 
This question can be further divided into two subcategories. One is to calibrate the mechanical error between the lidar and the rotating unit [1,4-15]. Due to the mechanical error, the relative position and attitude between the $2 \mathrm{D}$ lidar and rotating unit are not exactly the same as expected. The error is 6-degrees of freedom (DOF), containing a 3-DOF translation and 3-DOF rotation [4]. The mechanical error can decrease the accuracy of the 3D point cloud in many aspects, including the shape and attitude of the 3D point cloud. The mechanical error is inevitable because machining and assembling errors cannot be eliminated completely. Therefore, calibration is necessary.

The other subcategory is to calibrate the matching error between the $2 \mathrm{D}$ lidar and rotating unit (that is, the motor) [2,3,5,8,16-23]. The working principle of a rotating 2D lidar is to calculate the 3D coordinates of the points by using the data collected by the 2D lidar and the angle of the motor shaft. Therefore, in order to improve the accuracy of the $3 \mathrm{D}$ point cloud, it is necessary to match the data from the 2D lidar and the angle of the motor shaft accurately. If we can know the angle of the motor shaft corresponding to each point collected by the 2D lidar during the entire 3D scan of a rotating 2D lidar, the matching error between the 2D lidar and the motor can be eliminated. However, in reality, the situation mentioned above does not exist, so the matching error always exists, which has a negative impact on the accuracy of the 3D point cloud, both in shape and attitude.

The first subcategory focuses on the calibration of the mechanical error, and the second subcategory focuses on the calibration of the matching error. They are juxtaposed with each other and cannot be replaced by each other. Only when both the mechanical error and the matching error are calibrated, a rotating $2 \mathrm{D}$ lidar can get an accurate $3 \mathrm{D}$ point cloud in a static environment.

The first subcategory, the calibration method of the installation error between the 2D lidar and the rotating unit, has been thoroughly evaluated. For example, the calibration processes proposed in $[1,4,5]$ can work effectively without the need of specific scene, additional equipment, and a priori information about the environment (such as size etc.). The requirement is that there should be at least one plane in the scanning field. Planar features are abundant in man-made indoor environments, such as walls, floors, and ceilings.

However, the research on the second subcategory is relatively unexplored. The existing methods used for calibration of the matching error between a 2D lidar and motor mostly utilize expensive servo motors or encoders to output the motor rotation angle with the time stamps, and align it with the data form 2D data with time stamps. In this way, the matching error is calibrated $[2,3,5,8,16-23]$. Although for installation error, which is mentioned in the first subcategory, the calibration can be done at zero cost, without the need of additional equipment and all the work can be accomplished in normal indoor environment, while the calibration of the matching error is still expensive, for a servo motor or encoder is needed. Therefore, it is worthwhile to figure out a way to calibrate the matching error at low cost.

For the calibration of the matching error, the commonly used method is to align the data from the 2D lidar and the angle of the motor shaft by time stamps. Since the frequency of the two types of data is different, interpolation is used to obtain matched data pairs. The prerequisite for using this method is that the rotating unit that rotates the 2D lidar can output the absolute angle position of its shaft, which requires expensive device such as a servo motor or encode. This method has been used in [3,5,16-19]. A similar method was used in $[2,8,20]$, for each time, when a group of data from the $2 \mathrm{D}$ lidar is received, the angle of the motor shaft at this moment is recorded. According to the serial number of each data in this group, its corresponding motor shaft angle can be estimated linearly. In [21], a pitching 2D lidar was built. In this work, a high-precision potentiometer was used to measure the angle of the motor shaft. In [22], a rotating 2D lidar used on an unmanned aerial vehicle (UAV) was built, and the software package Spin_Hokuyo [23] was used to generate the 3D point clouds in real time. The applicable hardware for Spin_Hokuyo is a 2D lidar UTM-30LX Hokuyo and a servo motor Dynamixel MX-28, while the servo motor Dynamixel MX-28 also can output the rotation angle. In order to facilitate the use of this software package, the above hardware was used in [22]. In addition to the above literature, 
the rotating units in $[9,29]$ were stepper motors, without using encoders. The matching error was neglected, the matching between the 2D lidar and the motor was done through rough estimation, merely.

According to [2,3,5,8,16-23], in order to accurately match the 2D lidar and the motor, the rotating unit must be able to output the absolute angle position of the motor shaft. Therefore, a device like a servo motor or encoder is necessary. This goes against the idea of reducing costs. The reason why we built a rotating $2 \mathrm{D}$ lidar instead of using a commercial $3 \mathrm{D}$ lidar directly is because we wanted to reduce costs. The high price of commercial 3D lidars makes them unaffordable for many users, and the cost of a rotating 2D lidar is one-tenth of it. The obvious advantage of a rotating 2D lidar over a commercialized 3D lidar is the lower cost. Therefore, for a rotating 2D lidar, the question of how to further reduce its cost under the premise of ensuring accuracy is worth studying.

If a rotating 2D lidar is not equipped with an encoder or a servo motor, and only a stepper motor is used as the rotating unit, the cost can be further reduced. However, the angle of the motor shaft cannot be measured, because of which, the 2D lidar and the motor cannot be accurately matched according to the existing methods. This will reduce the accuracy of the 3D point cloud.

In this paper, we focus on the low-cost calibration of matching error between the lidar and the motor for a rotating 2D lidar. Compared with the existing methods, our method reduces the cost. In order to reduce the cost, we did not use a sensor to measure the angle of the motor shaft in our prototype, because it is expensive. We calibrated the matching error between the 2D lidar and the motor without using a servo motor or encoder. A stepper motor was used to rotate the 2D lidar, photoelectric switch and shading sheet were used to define the initial position of the motor shaft, a triangular plate was used to calibrate the matching error between the 2D lidar and the motor. While reducing the cost of a rotating 3D lidar, our method ensured the accuracy of the 3D point cloud. Our method provides a new idea for the further cost reduction of a rotating $2 \mathrm{D}$ lidar.

The rest of this paper is structured as follows. Section 2 describes the problem to be solved in this paper; the modeling of the system is described in Section 3. The calibration method is described in detail in Section 4. Then, we verify our method by experiments in Section 5. Finally, a brief conclusion and future work are given in Section 6.

The notations used throughout this paper have been listed in Table A1, which is in Appendix A.

\section{Problems}

According to the previous description, our goal is to use only a stepper motor to rotate a 2D lidar without using encoder or servo motor to reduce the cost under the premise of ensuring the accuracy of the 3D point cloud. For this purpose, a method is proposed in [2], an open-loop controlled stepper motor is used to rotate the 2D lidar, the motor stops rotating when its shaft turns to a certain position, and then the $2 \mathrm{D}$ lidar begin its scan. After the 2D lidar finishes scanning, the motor shaft rotates to the next position, and the 2D lidar scans again. This cycle continues until the 3D point cloud collection is completed. There is a certain angle between every two adjacent positions of the motor shaft, which determines the density of the 3D point cloud. This method attempts to align the data from the $2 \mathrm{D}$ lidar and the angle of the motor shaft at the cost of low efficiency, but it has obvious disadvantages. The frequent stop and start of the motor may cause mechanical failure. The acceleration and deceleration of the motor can decrease the accuracy of the rotation positioning. Due to the need of stopping the rotation of the motor shaft for multiple times, the time used for finishing a 3D scan will be prolonged. In view of the above disadvantages, it is necessary to develop a method to calibrate the matching error between 2D lidar and motor while the motor rotates continuously.

In our work, during the 3D scan process of a rotating 2D lidar, the 2D lidar scans continuously, and the shaft of the stepper motor rotates at a constant angular velocity. The matching between the two can no longer be done with the help of time stamps, because 
the stepper motor is open-loop, and there is no sensor to measure the rotation angle of the motor shaft, so the data of motor angle with time stamps cannot be obtained.

In this case, we initially tried to match the $2 \mathrm{D}$ lidar and the motor in the following way. After the angular resolution $\gamma$ in the direction of motor shaft rotation was configured, the number of scanning cycles $n$ of the 2D lidar in one 3D scan could be calculated as:

$$
n=\frac{180^{\circ}}{\gamma}
$$

The scanning frequency $f$ of the $2 \mathrm{D}$ lidar could be found in its product manual, so the time $T$ used for finishing a 3D scan of a rotating 2D lidar could be calculated as:

$$
T=\frac{n}{f}
$$

For a rotating 2D lidar, a 3D scan requires the motor shaft to rotate 180 degrees [2], so the angular velocity $\omega$ of the motor shaft could be calculated as:

$$
\omega=\frac{180^{\circ}}{T}
$$

Until now, the control parameters of the 2D lidar and the stepper motor have been calculated, the working time of the $2 \mathrm{D}$ lidar in continuous scanning mode is $T$, the shaft of the stepper motor rotates at angular velocity $\omega$, and its working time is $T$. We made them start working at the same time, so that ideally they could be matched.

However, we found that there are obvious errors in the 3D point cloud, both in the shape and attitude. Additionally, for each 3D scan, the error is not constant. Our experiment is shown in Figure 1, Figure 1a is a photo of the environment for collecting 3D point cloud. The errors we observed are shown in Figure 1b,c. The environment is a conference room. The position of the rotating 2D lidar is aligned with the gap between the tiles on the ground to ensure that it is not skewed relative to the conference room. In this way, the top view of the 3D point cloud should be correct in shape and attitude, and coincide with the blue wireframe. This is not actually the case. The shape is distorted and the attitude is skewed. In addition, for each $3 \mathrm{D}$ scan, the error of the $3 \mathrm{D}$ point cloud is uncertain, and the $3 \mathrm{D}$ point cloud may appear as in Figure 1b,c.

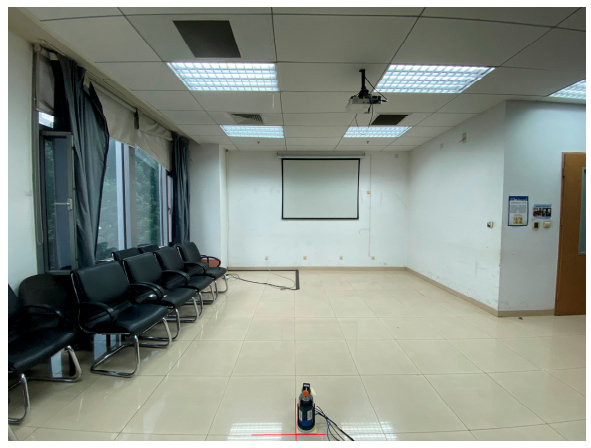

(a)

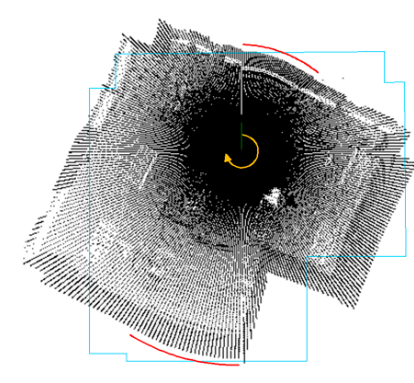

(b)

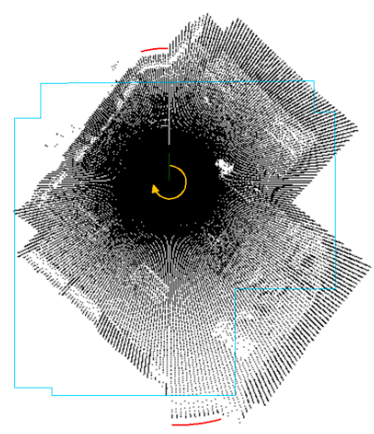

(c)

Figure 1. The shape error and attitude error of the 3D point cloud: (a) a photo of the environment for collecting the 3D point cloud; (b) top view of a 3D point cloud collected by a rotating 2D lidar; (c) top view of another 3D point cloud collected by a rotating 2D lidar.

There are several reasons for the error.

(1) As mentioned in [1,4-10], the mechanical error between the 2D lidar and rotating unit can cause distortion of the 3D point cloud. Based on the idea of control variables, in order to focus on other reasons, we need to exclude this item first. By improving the 
manufacture and assembly accuracy of the mechanical parts of our prototype, we minimized the impact of mechanical errors on the accuracy of the 3D point cloud. We used computer numerical control (CNC) machine tools to manufacture the key parts of our prototype, with a tolerance level of IT5 (Chinese standard, GB/T1184-1996). In Appendix B, we verified that the self-reasons of our prototype (which include the mechanical error) has a much smaller impact on the accuracy of the 3D point clouds than the error of the 2D lidar itself $( \pm 40 \mathrm{~mm})$. Therefore, we can exclude this item and assume that other reasons are causing the resulting observed error.

(2) The acceleration and deceleration of the motor shaft will cause error. In a 3D scan of a rotating 2D lidar, we assume roughly that the shaft of the stepper motor rotates at angular velocity $\omega$ for time $T$, and it rotates $180^{\circ}$ in total. However, in practice, the motion of the motor shaft is more complex. The motor shaft accelerates from standstill to angular velocity $\omega$, and then it keeps rotating at this speed, after which it decelerates to standstill. We have ignored the acceleration and deceleration of the motor shaft, and this can lead to the shape error of the 3D point cloud, which is especially obvious at the closed position of the point cloud. The so-called closed position here refers to the area where the points collected by the 2D lidar when the motor shaft starts to rotate or stops rotating. The areas of these two types of points are adjacent, and these points are most directly affected by the acceleration and deceleration of the motor shaft.

(3) The use of photoelectric switches may cause error. Since no encoder or servo motor is used, it is necessary to define the initial position of motor shaft by photoelectric switch. When finding the initial position, the motor shaft will rotate back and forth by a large margin and make the shading sheet which rotates with the motor shaft triggering the photoelectric switch. After the photoelectric switch is triggered, the controller sends a stop command to the motor to stop its shaft. The position of the motor shaft after it stops is considered to be the initial position. This process can be described in more detail, the shading sheet blocks the light beam of the photoelectric switch - the photoelectric switch is triggered - the controller sends the stop command to the stepper motor-the shaft of stepper motor decelerates until it stops. The accuracy of the initial position of the motor shaft is affected by many factors such as the response time of the photoelectric switch, the rotation direction and the speed of the motor shaft. For each 3D scan of a rotating 2D lidar, the motor shaft starts rotating from its initial position. Therefore, if the initial position of the motor shaft is not accurately defined, the attitude error of the $3 \mathrm{D}$ point cloud will be caused.

(4) The uncertain time deviation may cause error. In the actual engineering operation, we found that although we require the 2D lidar and the stepper motor to start to work at the same time, there is an uncertain time deviation between the starting time of the 2D lidar and the starting time of the stepper motor. The value of this time deviation is very small, but it is enough to have a significant impact on the accuracy of the 3D point cloud. The reason for the uncertain time deviation is that the response time of the 2D lidar and stepper motor to the command is inconsistent and not constant, as mentioned in [16]. Because both the transmission of the command and the response of the motor or the 2D lidar take time, there is an uncertain time deviation between the time when the controller starts to send the command to the motor and the time when the motor starts to work. Similarly, there is an uncertain time deviation between the time when the controller starts to send the command to the 2D lidar and the time when the 2D lidar starts to work. The uncertainty of the transmission time of the command and the response time of the device is a common problem which is difficult to solve. Because of this problem, we can mark the time when the controller starts to send the command to the motor or the 2D lidar in the program, we cannot know the actual working time of the motor or $2 \mathrm{D}$ lidar. This is the cause of the uncertain time deviation between the starting time of the 2D lidar and the starting time of stepper motor. For each $3 \mathrm{D}$ scan of a rotating $2 \mathrm{D}$ lidar, this time deviation is uncertain, so 
the shape and attitude error of the 3D point cloud for each scan is also uncertain (see Figure 1). The reason for the shape error of the 3D point cloud can be analyzed as follows. Due to the uncertain time deviation, there may be two situations. One is that the motor starts to rotate after the 2D lidar has started scanning continuously for a short period of time. The 3D point cloud collected in this case is shown in Figure 1b. At the position where the motor starts to rotate (the beginning of the yellow circular arrow), there is a shape error marked by red line. The other situation is that the motor starts working earlier than the 2D lidar, so that the motor has stopped rotating before the 2D lidar stops scanning. The 3D point cloud collected in this case is shown in Figure 1c. At the position where the motor stops rotating (the end of the yellow circular arrow), there is a shape error marked by red line. In either case, a shape error of the 3D point cloud will result. In addition, the attitude error can also be caused. Therefore, the uncertain time deviation between the starting time of the 2D lidar and the starting time of stepper motor is one of the reasons for the uncertain error of the 3D point cloud in shape and attitude.

In addition to item 1 of the above 4 items, it is our work to exclude the errors in the other three items. Unfortunately, these three errors mentioned above may be uncertain, for the acceleration and deceleration curve of the motor shaft may be uncertain, the response time of the photoelectric switch may be uncertain, especially, the time deviation between the moment when the 2D lidar starts to work and the moment when stepper motor starts to work may be uncertain, too. Due to the above uncertainties, the shape and attitude error of the 3D point clouds may also be uncertain.

The effect of item 2, 3 and 4 on the 3D point cloud can be divided into two categories, one is shape error and the other is attitude error, as shown in Figure 1. For simplicity, we first only analyze the shape error of the 3D point cloud.

According to Formulas (1)-(3), we can find that if the shape of the 3D point cloud is correct, the necessary and sufficient condition is that the stepper motor rotates at constant angular velocity $\omega$ during the continuous scanning of the 2D lidar. The acceleration and deceleration of motor shaft in item 2 will cause the shape error of the 3D point cloud. We have tried to estimate the approximate speed curve of the motor shaft, and to eliminate the shape error of the 3D point cloud. It turns out that this is not feasible. First, the acceleration and deceleration curve of the motor shaft may not be constant. Second, the shape error of the $3 \mathrm{D}$ point cloud is not entirely attributed to the acceleration and deceleration of motor shaft. The uncertain time deviation in item 4 also has a significant impact on the shape of the 3D point cloud. Therefore, the shape error of 3D point cloud cannot be effectively eliminated by estimating the approximate speed curve of the motor shaft.

If the action sequence of the 2D lidar and the stepper motor is modified, making the motor start to work earlier and stop working later than the 2D lidar, the acceleration and deceleration of motor shaft will be staggered with the working time of the 2D lidar, as shown in Figure 2. In this way, before the continuous scanning of the 2D lidar, the shaft of the stepper motor has been accelerated and rotates at constant angular velocity $\omega$. After the 2D lidar stops scanning, the stepper motor starts to decelerate. During the continuous scanning of the $2 \mathrm{D}$ lidar, the stepper motor rotates $180^{\circ}$ at constant angular velocity $\omega$. The advantage of the modified action sequence is that the factors causing the shape error of the $3 \mathrm{D}$ point cloud in item 2 and item 4 can be excluded, and the 3D point cloud with correct shape can be obtained.

Through the above method, the uncertain shape error of the 3D point cloud can be eliminated, but the uncertain attitude error of the 3D point cloud is still remains to be solved. Due to the error of the photoelectric switch (which is mentioned in item 3), it is impossible to accurately define the initial position of the motor shaft. Due to the uncertainty of the acceleration and deceleration curve (which is mentioned in item 2) and the uncertain time deviation between the starting time of the 2D lidar and the starting time of the motor (which is mentioned in item 4), it is impossible to know how much angular rotation the motor shaft has rotated at the moment the 2D lidar starts to work. Therefore, it is impossible 
to know the position of the motor shaft when a rotating 2D lidar starts a 3D scan. This will cause the attitude error of the 3D point cloud, and the error is uncertain. Due to the uncertainty of the error, we cannot calibrate it in general way by finding a constant to offset it.

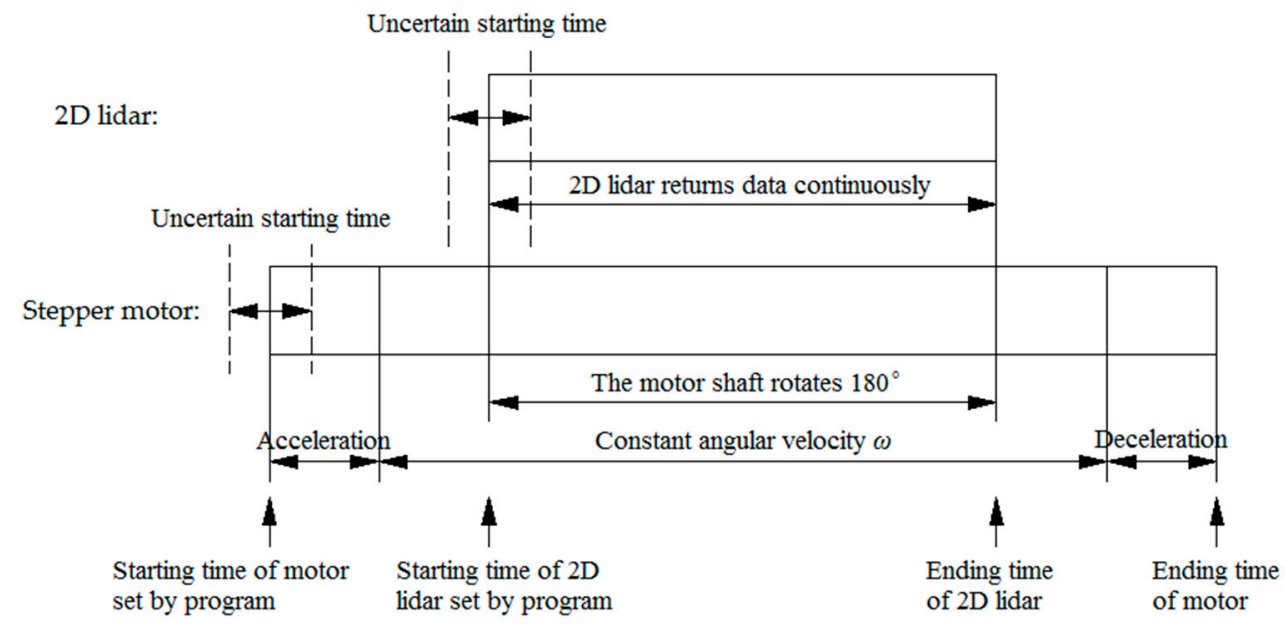

Figure 2. Improved action sequence of the 2D lidar and stepper motor.

Generally speaking, for the calibration of error, if the error to be calibrated is constant (such as mechanical installation error), the calibration of this error will be relatively easy. Because the mechanical installation error is constant, unless the mechanical parts are reinstalled. In order to calibrate this kind of error, we can find a constant estimated value for it.

In our research, the error to be calibrated is not constant. This increases the difficulty of the calibration of this error. For each 3D scan of our prototype, the true value of this error is different. We cannot find a constant estimated value for it according to the conventional calibration methods.

As for why this error is uncertain, we have already analyzed the reasons above. Some factors include some uncertainties (such as uncertain motor acceleration and deceleration curve, uncertain response time of photoelectric switch, uncertain time deviation between the starting time of the 2D lidar and the starting time of the stepper motor), and the combination of these uncertainties leads to the uncertainty of this error. Because this error is shown on the attitude of the 3D point cloud, therefore, we call it uncertain attitude error in our paper.

The calibration of the uncertain attitude error of the 3D point cloud is the focus of this paper. To solve this problem, a triangular plate is used as a reference object, which is installed on the rotating unit as part of the prototype. We recognize the corresponding part of the triangular plate in the scanned 3D point cloud, and correct the attitude of the 3D point cloud according to the known position of the triangular plate.

\section{System Modeling}

\subsection{Overview of Prototype}

The prototype we built is simple. A 2D lidar Hokuyo UST-10LX [30] is used to scan the environment, its angle resolution is $0.25^{\circ}$. A 42 -stepper motor with its driver and controller are used to rotate the $2 \mathrm{D}$ lidar. A photoelectric switch is used for the definition of the initial position of the motor shaft. In addition to the above-mentioned electronic devices, there are also some mechanical parts. A connector is used for it is necessary to fix the 2D lidar on the motor shaft, and sufficient installation accuracy should be ensured. A shading sheet which rotates with the motor shaft is required to block the light beam of the photoelectric switch so as to trigger it. A triangular plate is needed to correct the attitude of the 3D point cloud. The photo of our prototype is shown in Figure 3. 


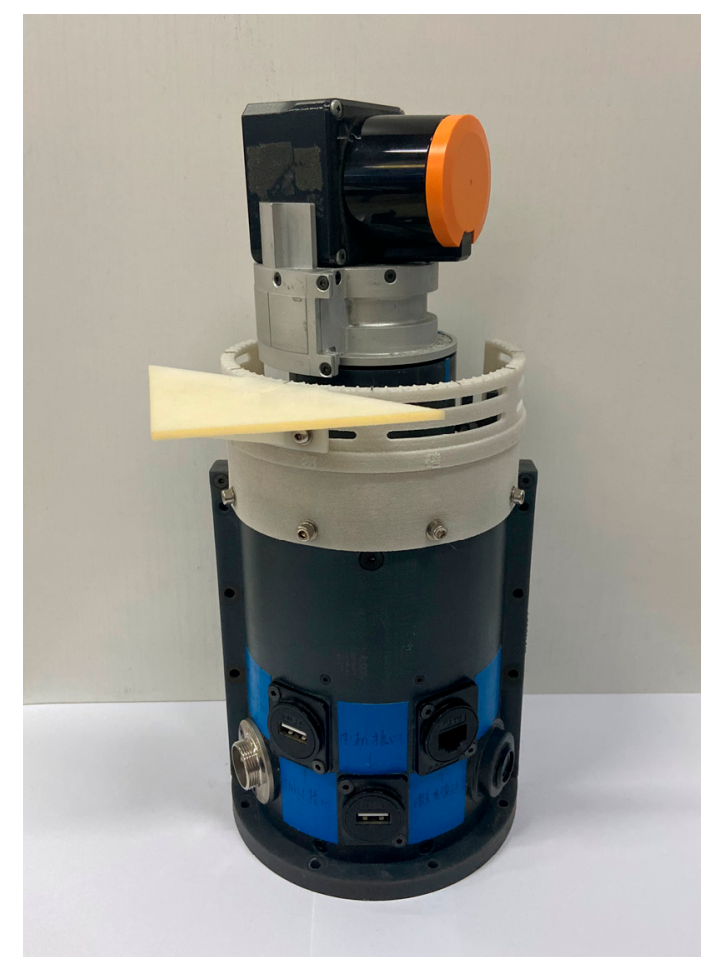

Figure 3. A photo of our prototype. The 2D lidar Hokuyo UST-10LX is installed on a rotating unit, and a triangular plate is used to calibrate the attitude error of the $3 \mathrm{D}$ point cloud.

In some prototypes mentioned by several literatures (such as [8]), a slip ring is used to connect the rotating end (the 2D lidar) and the stationary end (the stationary part of the rotating unit) of a rotating $2 \mathrm{D}$ lidar in power supply and communication. Under the action of the slip ring, the rotating cables of the $2 \mathrm{D}$ lidar is transformed into stationary cables. By using a slip ring, the motor can rotate the 2D laser continuously without twisting or pulling the cable. However, the use of a slip ring can also lead to an increase of weight, size and cost. Therefore, we have not used a slip ring in our prototype, as a result of which, we need to pay attention to the layout of the cable to ensure that the cable cannot be pulled when the motor shaft rotates in a 3D scan. After each 3D scan, it is necessary to return the motor shaft to the original position, because due to the limitation of the cable, the motor can only rotate in a certain range and cannot rotate continuously.

According to the different angular resolution in motor shaft direction, our prototype has 7 scanning modes. In mode 1, the angular resolution is lowest, and it takes only $1 \mathrm{~s}$ to finish a 3D scan, which is suitable for applications with high real-time requirement. In mode 7 , the angular resolution is highest, and it takes $16 \mathrm{~s}$ to finish a 3D scan. In this mode, dense point cloud with meticulous details of the environment can be collected. These 7 scanning modes make our prototype suitable for different applications. The time $T$ and the angular resolution $\gamma$ of each mode are shown in Table 1. The relationship between $T$ and $\gamma$ is can be found in Equations (1) and (2).

Table 1. Seven scanning modes of our prototype.

\begin{tabular}{cccccccc}
\hline Mode & $\mathbf{1}$ & $\mathbf{2}$ & $\mathbf{3}$ & $\mathbf{4}$ & $\mathbf{5}$ & $\mathbf{6}$ & $\mathbf{7}$ \\
\hline$T / \mathrm{s}$ & 1 & 2 & 4 & 5 & 8 & 10 & 16 \\
$\gamma /{ }^{\circ}$ & 4.5 & 2.25 & 1.125 & 0.9 & 0.5625 & 0.45 & 0.28125 \\
\hline
\end{tabular}

\subsection{Coordinate Conversion}

The principle of our prototype is to combine the data collected by the 2D lidar with the rotation angle of the motor shaft, and then calculate the $3 \mathrm{D}$ coordinates of the sampling 
points. After that, the attitude of the point cloud is corrected according to the known position of the triangular plate. This process involves the conversion among three Cartesian coordinate frames, namely the coordinate frame of the 2D lidar $(L)$, the coordinate frame of rotating unit before attitude correction $\left(\mathrm{O}^{\prime}\right)$ and the coordinate frame of rotating unit after attitude correction $(O)$, as shown in Figure 4.

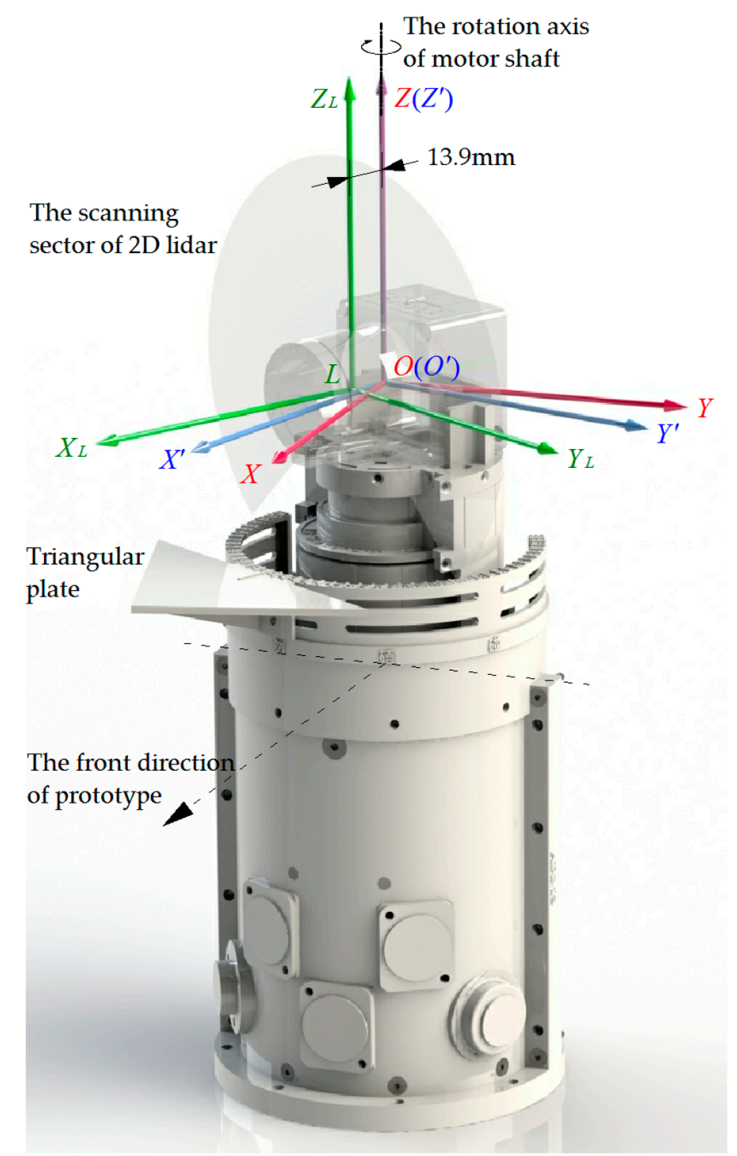

Figure 4. Three Cartesian coordinate frames. The coordinate frame of the $2 \mathrm{D}$ lidar $(L)$ is shown in green, the coordinate frame of rotating unit before attitude correction $\left(O^{\prime}\right)$ is shown in blue, and the coordinate frame of rotating unit after attitude correction $(O)$ is shown in red.

The definition of the $2 \mathrm{D}$ lidar coordinate frame $L-X_{L} Y_{L} Z_{L}$ is as follows. The origin point $L$ of this coordinate frame is the center of the $2 \mathrm{D}$ lidar scanning sector. The plane $Y_{L} L Z_{L}$ is coplanar with the scanning sector. The axis $Z_{L}$ is the middle line of the scanning sector, and the axis $X_{L}$ is perpendicular to the scanning sector. The position of coordinate frame $L$ relative to the $2 \mathrm{D}$ lidar is fixed, but its position relative to rotating unit is not fixed, because the $2 \mathrm{D}$ lidar is rotational relative to rotating unit.

The definition of coordinate frame $O^{\prime}-X^{\prime} Y^{\prime} Z^{\prime}$ is as follows. This is the coordinate frame of the rotating unit before attitude correction. The origin point $O^{\prime}$ of the coordinate frame is located on the rotating axis of the motor shaft, and the line composed of point $L$ and point $O^{\prime}$ is perpendicular to the rotating axis of the motor shaft. The axis $Z^{\prime}$ coincides with the rotation axis of the motor shaft and is parallel to the axis $Z_{L}$ of coordinate frame $L$. The axis $Y^{\prime}$ coincides with the starting and ending positions of a 3D scan, as shown in Figure 5. Since the starting and ending positions of a 3D scan is uncertain (as described in Section 2), the position of coordinate frame $O^{\prime}$ relative to the rotating unit is uncertain, too.

The definition of coordinate frame $O-X Y Z$ is as follows. This is the coordinate frame of the rotating unit after attitude correction, its origin point $O$ coincides with the origin point $O^{\prime}$ of coordinate frame $O^{\prime}-X^{\prime} Y^{\prime} Z^{\prime}$. Its axis $Z$ coincides with the axis $Z^{\prime}$ of coordinate frame $O^{\prime}-X^{\prime} Y^{\prime} Z^{\prime}$. Although the position of coordinate frame $O^{\prime}$ relative to the rotating unit 
is uncertain, the position of coordinate frame $O$ relative to the rotating unit is certain, and the positive direction of axis $X$ parallel to the front direction of the prototype (as shown in Figure 4).
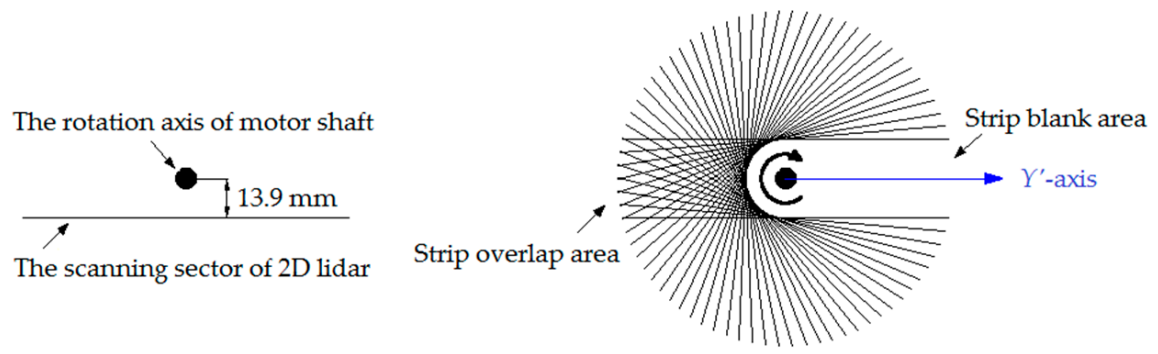

Figure 5. The scanning sector of the $2 \mathrm{D}$ lidar does not coincide with the rotation axis of the motor shaft. They are parallel to each other, and the distance between them is $13.9 \mathrm{~mm}$. This design can collect the 3D point cloud with a mark, which can roughly show the position of the motor shaft at the moment a $3 \mathrm{D}$ scan is started, that is, the position of the $Y^{\prime}$-axis of coordinate frame $O^{\prime}-X^{\prime} Y^{\prime} Z^{\prime}$.

In our prototype, the scanning sector of the 2D lidar does not coincide with the rotation axis of the motor shaft, and they are parallel to each other, the distance between them is $13.9 \mathrm{~mm}$ (as shown in Figure 4). The reason for this design can be explained in Figure 5. Through this design, we can collect the 3D point cloud with strip blank area and strip overlap area (from the top view). This is an important mark. The parts of the 3D point cloud on both sides of this mark are collected when the rotating 2D lidar starts or stops a 3D scan. This mark can roughly show the position of the motor shaft at the moment a $3 \mathrm{D}$ scan is started, that is, the position of the axis $Y^{\prime}$ of coordinate frame $O^{\prime}-X^{\prime} Y^{\prime} Z^{\prime}$. We can find that for each $3 \mathrm{D}$ scan, the position of this mark relative to the $3 \mathrm{D}$ point cloud is not constant. For more details, see the experiment section in Section 5.

After introducing the definitions of the above three Cartesian coordinate frames, the conversion among them should be studied out. Our goal is to get 3D point cloud relative to coordinate frame $O$ accurately, both in shape and attitude. Our raw data includes ranging data $r$ and azimuth angle $\theta$, which is obtained from the 2D lidar, and the rotation angle $\varphi_{\text {motor }}$ of the motor shaft. Among them, $r$ can be obtained directly, while $\theta$ and $\varphi_{\text {motor }}$ can be obtained by linear interpolation.

First, we calculate a $2 \mathrm{D}$ point $p_{L}$ relative to coordinate frame $L$ according to the ranging data $r$ and azimuth angle $\theta$

$$
p_{L}=r\left[\begin{array}{lll}
0 & s(\theta) & c(\theta)
\end{array}\right]^{T}
$$

where $c(\cdot)$ and $s(\cdot)$ are cos and sin, respectively.

Second, we convert point $\boldsymbol{p}_{L}$ in coordinate frame $L$ to the corresponding point $\boldsymbol{p}_{\mathrm{O}^{\prime}}$ in coordinate frame $O^{\prime}$

$$
\boldsymbol{p}_{\mathrm{O}^{\prime}}=\boldsymbol{R}_{M}\left(R_{L}^{O^{\prime}} \boldsymbol{p}_{L}+\boldsymbol{t}_{L}^{\mathrm{O}^{\prime}}\right)
$$

where $\boldsymbol{R}_{L}^{O^{\prime}}$ and $\boldsymbol{t}_{L}^{O^{\prime}}$ are the rotation matrix and the translation vector from coordinate frame $L$ to coordinate frame $O^{\prime}$ at the moment when a 3D scan is started, respectively. At this moment, axis $X_{L}$ coincides with axis $X^{\prime}$, axis $Y_{L}$ and axis $Y^{\prime}$ are parallel, axis $Z_{L}$ and axis $Z^{\prime}$ are parallel, and the distance between point $L$ and point $O^{\prime}$ is $13.9 \mathrm{~mm}$. Therefore, We can know that $\boldsymbol{R}_{L}^{O^{\prime}}=\boldsymbol{I}, \boldsymbol{t}_{L}^{O^{\prime}}=(13.9 \mathrm{~mm} 00)^{T} . \boldsymbol{R}_{\mathrm{M}}$ is the rotation matrix calculated according to the rotation angle $\varphi_{\text {motor }}$ of the motor shaft, which shows the attitude change of coordinate frame $L$ relative to its initial position after being rotated by the motor shaft. Since the direction of the rotation is along the axis $Z^{\prime}$, there is no rotation component in other directions, $\boldsymbol{R}_{\mathrm{M}}$ can be calculated as:

$$
\boldsymbol{R}_{M}=\left[\begin{array}{ccc}
c\left(\varphi_{\text {motor }}\right) & -s\left(\varphi_{\text {motor }}\right) & 0 \\
s\left(\varphi_{\text {motor }}\right) & c\left(\varphi_{\text {motor }}\right) & 0 \\
0 & 0 & 1
\end{array}\right]
$$


where $\varphi_{\text {motor }}$ is calculated linearly according to the serial number of the corresponding point in all points. The total number of points in the point cloud obtained by a 3D scan of a rotating $2 \mathrm{D}$ lidar is denoted as $\mathrm{K}$, the rotation angle of the motor shaft at the $k$-th point is:

$$
\varphi_{\text {motor }}^{k}=\frac{k}{\mathrm{~K}} \times 180^{\circ}
$$

Until now, we can get the $3 \mathrm{D}$ point cloud relative to coordinate frame $O^{\prime}$, which has an uncertain attitude error, but no shape error. The calibration of this uncertain attitude error is the next step.

Third, we convert point $\boldsymbol{p}_{O^{\prime}}$ in coordinate frame $O^{\prime}$ to the corresponding point $\boldsymbol{p}_{O}$ in coordinate frame $O$

$$
\boldsymbol{p}_{O}=\boldsymbol{R}_{\mathrm{O}^{\prime}}^{O} \boldsymbol{p}_{\mathrm{O}^{\prime}}
$$

where $R_{O^{\prime}}^{O}$ is the rotation matrix from coordinate frame $O^{\prime}$ to coordinate frame $O$. Since point $O^{\prime}$ and point $O$ are coincident, the translation vector from coordinate frame $O^{\prime}$ to coordinate frame $O$ is a zero vector. $R_{O^{\prime}}^{O}$ is calculated according to the angle $\varphi_{\text {offset }}$, while $\varphi_{\text {offset }}$ shows the deflection angle of the coordinate frame $O^{\prime}$ relative to the coordinate frame $O$ in the $Z$-axis direction. Since the direction of the deflection is along the axis $Z^{\prime}$, there is no rotation component in other directions, $\boldsymbol{R}_{O^{\prime}}^{O}$ can be calculated as:

$$
\boldsymbol{R}_{\mathrm{O}^{\prime}}^{O}=\left[\begin{array}{ccc}
c\left(\varphi_{\text {offset }}\right) & -s\left(\varphi_{\text {offset }}\right) & 0 \\
s\left(\varphi_{\text {offset }}\right) & c\left(\varphi_{\text {offset }}\right) & 0 \\
0 & 0 & 1
\end{array}\right]
$$

The remaining problem is to calculate the angle $\varphi_{\text {offset }}$. According to the above description, it can be known that for each 3D scan of a rotating 2D lidar, the value of angle $\varphi_{\text {offset }}$ is not constant. If we can accurately calculate the value of $\varphi_{\text {offset }}$ for each 3D scan, then we can eliminate the uncertain attitude error of the 3D point cloud according to Equations (8) and (9). As a result of which, we can get the 3D point cloud relative to coordinate frame $O$ accurately-both in shape and attitude.

The calculation method of the angle $\varphi_{\text {offset }}$ for each 3D scan will be described in more detail in the next section.

\section{Method}

\subsection{Calibration of Uncertain Attitude Error of 3D Point Cloud}

According to Equations (4)-(7), we have obtained the 3D point cloud with correct shape relative to the coordinate frame $O^{\prime}$, which can be called point cloud $C_{O^{\prime}}$. Next, we will correct the attitude error of point cloud $C_{O^{\prime}}$ according to the value of the angle $\varphi_{\text {offset }}$. The angle $\varphi_{\text {offset }}$ is calculated as follows.

In the first step, the point cloud corresponding to the triangular plate (which is called $C_{\text {tri }}$ ) should be extracted from the point cloud $C_{O^{\prime}}$. We define a special area (the blue area in Figure 6), and the point cloud in this area is the point cloud $\boldsymbol{C}_{\text {tri }}$, which corresponds to the triangular plate, because there is only the triangular plate in this area and no other objects exist.

The blue area in Figure 6 is a semi-ring. Its inner radius is $62.5 \mathrm{~mm}$, outer radius is $115.5 \mathrm{~mm}$ and the thickness is $20 \mathrm{~mm}$. The cut surface of the half ring is aligned with the axis $Y^{\prime}$ of the coordinate frame $O^{\prime}$, and the axis of the half ring coincides with the rotation axis of motor shaft. There is only the triangular plate in this area and no other objects are allowed to exist, otherwise, the extracted point cloud will not only be the point cloud corresponding to the triangular plate, it will be mixed with the point clouds of other objects. Therefore, we need to pay attention to the following two points. First, there should be no other objects (such as cables) around the triangular plate, it may affect the extraction of the triangular plate. Second, as for the blue area, it is necessary to shrink its size as much as possible while ensuring that the triangular plate is fully included in it, this can reduce the probability of objects other than the triangular plate being included in the blue area. Since 
the position of the coordinate frame $O^{\prime}$ is uncertain relative to the rotating unit, the position of the triangular plate relative to the defined area is also uncertain, but this uncertainty is within a certain range. Therefore, as long as the central angle of the blue ring area is big enough, it can be ensured that the triangular plate can be completely included in it. In our work, the central angle is set to $180^{\circ}$, and then sufficient tests have been done. We found that in multiple tests of the 7 modes of our prototype, the triangular plate can be completely included in the defined blue area.
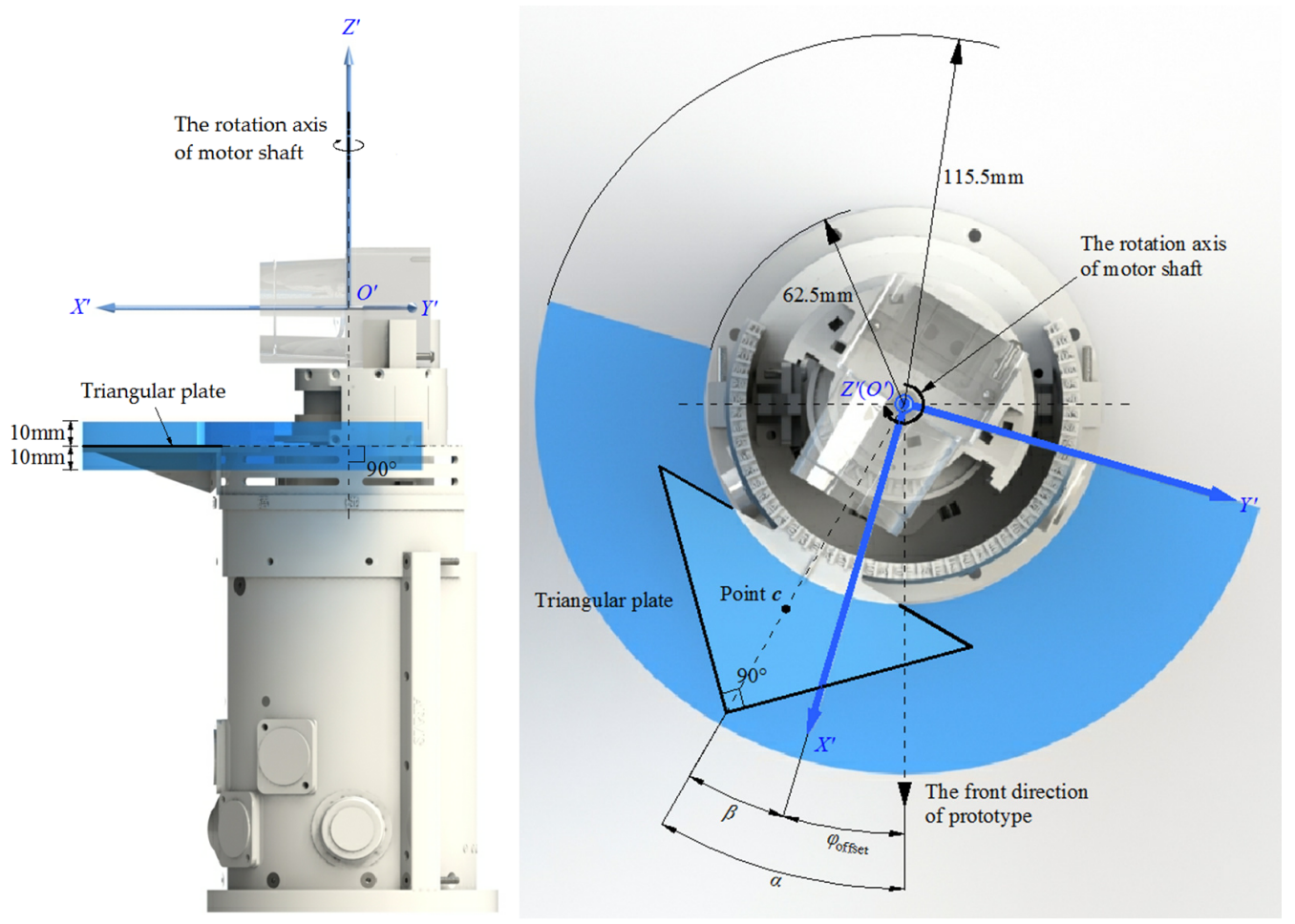

Figure 6. In order to extract the point cloud $C_{\text {tri }}$ from the point cloud $C_{O^{\prime}}$, we define a special area, which is shown in blue.

In the second step, the 3D coordinates of all the points in the point cloud $C_{\text {tri }}$ are averaged to calculate the center point $c$ of the triangular plate. This is done to find the middle line of the triangular plate, which is the perpendicular line from the point $c$ to the rotation axis of motor shaft. The angle $\alpha$ between the middle line and the front direction of the prototype (which is also the direction of $X$-axis direction of coordinate frame $O$ ) is known. It is a constant value, which depends on the installation position of the triangular plate on the prototype. The angle $\beta$ between the middle line and $X^{\prime}$-axis of coordinate frame $O^{\prime}$ can be calculated according to the 3D coordinates of the point $c$. According to the description above, the value of angle $\beta$ is uncertain. The angle between the $X$-axis of the coordinate frame $O$ and the $X^{\prime}$-axis of the coordinate frame $O^{\prime}$ is $\varphi_{\text {offset }}$. It can be seen from Figure 6 that the calculation formula for the angle $\varphi_{\text {offset }}$ is:

$$
\varphi_{\text {offset }}=\alpha-\beta
$$

Until now, we have calculated the deflection angle $\varphi_{\text {offset }}$ of the coordinate frame $O^{\prime}$ relative to the coordinate frame $O$ in the direction of $Z$-axis. Then, we can eliminate the uncertain attitude error of the 3D point cloud according to Equations (8) and (9). A point cloud relative to the coordinate frame $O$ can be obtained, this point cloud is called $C_{O}$. It is accurate both in shape and attitude.

A summary of the above process is as follows. Because of the uncertainty of the attitude error of the 3D point cloud, we cannot find a constant estimated value for it according to the conventional calibration methods. When using a conventional method 
to calibrate the error, a constant estimated value of the error will be found out, and it is used to correct the adverse effects of the error. However, when the value of the error is not constant, the conventional method will fail.

To calibrate this uncertain attitude error, we use a triangular plate. It is fixedly installed on the prototype, so it is fixed relative to the coordinate frame $O-X Y Z$. We can know its attitude relative to the coordinate frame $O-X Y Z$ according to the installation position of the triangular plate on the prototype.

In addition, we can extract the point cloud $C_{\text {tri }}$ corresponding to the triangular plate from the point cloud $C_{O^{\prime}}$ and calculate its attitude relative to the point cloud $C_{O^{\prime}}$. Since the attitude of the triangular plate relative to the point cloud $C_{O^{\prime}}$ is known, and the attitude of the triangular plate relative to the coordinate frame $O-X Y Z$ is also known, therefore, the attitude of the point cloud $C_{O^{\prime}}$ relative to the coordinate frame $O-X Y Z$ can be calculated, that is, the attitude error of point cloud $C_{O^{\prime}}$ can be calculated. Here, the triangular plate plays a key role.

For each 3D scan of our prototype, we perform the above process to obtain the attitude error of the point cloud $C_{O^{\prime}}$ corresponding to this scan. Although the attitude error of the point cloud $C_{O^{\prime}}$ is not constant for each $3 \mathrm{D}$ scan, we can calculate it in each $3 \mathrm{D}$ scan. In this way, we can calibrate it.

The following items should be paid attention to when calculating the angle $\varphi_{\text {offset }}$ with the above method.

(a) In our method, we define the middle line of the triangular plate according to the center point $c$. We have also tried to define the middle line by finding the point in point cloud $C_{\text {tri }}$ which is farthest from the rotation axis of the motor shaft (that is, the right-angled vertex of the triangular plate), it turns out that it is less accurate. The reason is obvious, the center point $c$ is calculated based on all the points in point cloud $C_{\text {tri }}$, while the farthest point is just one point selected from point cloud $C_{\text {tri }}$. Therefore, the former is more accurate. Moreover, the accuracy of the 2D lidar Hokuyo UST-10LX we use in our prototype is $\pm 40 \mathrm{~mm}$. At this level of accuracy, it is necessary to use the average of multiple points instead of a single point to define the middle line of the triangular plate.

(b) The larger the triangular plate is, the more points are contained in the point cloud $\boldsymbol{C}_{\text {tri }}$, and the calculation of the center point $c$ is less affected by the accidental error. However, if the size of the triangular plate is too large, it will be inconvenient. The triangular plate installed on our prototype is an isosceles right triangle. The lengths of its three edges are $79.2 \mathrm{~mm}, 79.2 \mathrm{~mm}$ and $112 \mathrm{~mm}$ respectively.

(c) The distance between the triangular plate and the motor shaft should be moderate. If the distance is too close, a part of the triangular plate will be in the blind area of the scanning field and cannot be scanned. If the distance is too far, the beam of $2 \mathrm{D}$ lidar will irradiate the triangular plate at a more inclined angle, and the number of the points contained in the point cloud $C_{\text {tri }}$ will be reduced. In our prototype, the distance between the base edge of the isosceles right triangle and the rotation axis of the motor shaft is $54.75 \mathrm{~mm}$.

(d) Since the center point $c$ is calculated by averaging the 3D coordinates of all the points in the point cloud $C_{\text {tri }}$, the points in point cloud $C_{\text {tri }}$ should be evenly distributed on the surface of the triangular plate. Therefore, the strip blank area and strip overlap area shown in Figure 5 should be staggered with the point cloud $C_{\text {tri }}$. This should be considered when determining the installation position of the triangular plate on the prototype. The installation position determines the value of the angle $\alpha$. In our prototype, $\alpha=30^{\circ}$.

\subsection{Calibration of Installation Error of Triangular Plate}

In the actual tests, we found the following problems. Due to the installation error of the triangular plate on the prototype, the actual value of the angle $\alpha$ is inconsistent with the expected value (which is $30^{\circ}$ ), this will make point cloud $C_{O}$ still have an attitude 
error. Even seemingly trivial installation errors can result in obvious attitude error of point clouds $C_{O}$. Unlike the uncertain attitude error described above, the attitude error here is constant. In order to calibrate this error, we need to find the optimal estimated value $\alpha^{*}$. This is an optimization problem. Since this error originates from the installation error of the triangular plate, only one calibration is required for this error, unless the installation position of the triangular plate has been changed, that is, it has been removed and reinstalled. If the installation position of the triangular plate has been changed, the error needs to be calibrated again.

We need to quantify the attitude error of the point cloud $C_{O}$. Through the work described above, the point cloud $C_{O}$ is already known. We collect the 3D point cloud of the conference room once again according to the method described in Figure 1, and perform the following processing on the 3D point cloud. We extract the planes corresponding to the 4 walls of the room, and calculate their unit normal vectors $n_{1}, n_{2}, n_{3}, n_{4}$ (this can be done through Point Cloud Library [31]), as shown in Figure 7.

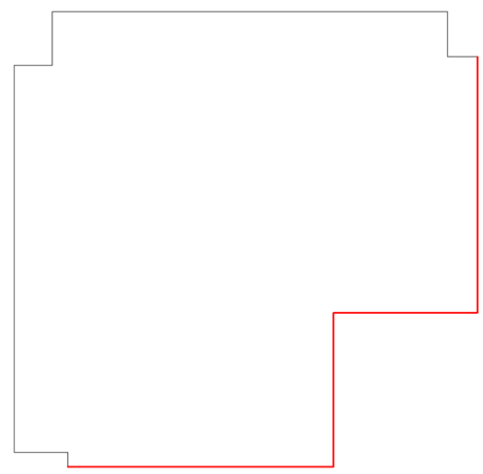

(a)

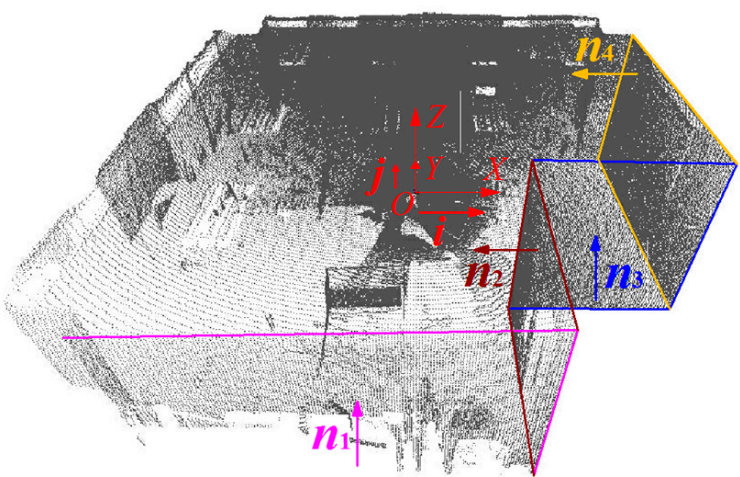

(b)

Figure 7. Extract the planes corresponding to the 4 walls of the conference room, and calculate their unit normal vector. (a) The top view of the room, where the red bold line is the walls used to extract the planes. Our selection criterion is as follows. The area of the walls should be large so as to facilitate the extraction of the planes. Besides, walls with windows should be avoided, because irregularly shaped curtains may interfere with the extraction of the planes; (b) the 3D point cloud of the conference room, from which we extracted the planes corresponding to 4 walls. We marked them with different colors to distinguish them. There are 6 vectors in $(b)$, where the vectors $n_{1}, n_{2}, n_{3}, n_{4}$ are the unit normal vectors of the 4 planes, and the vectors $i$ and $j$ are the unit normal vectors of $X$-axis and $Y$-axis of coordinate frame $O$.

Theoretically, the 3D point cloud in Figure 7 should not be skewed relative to the conference room. However, due to the installation error of the triangular plate, the actual value of the angle $\alpha$ is inconsistent with the expected value, resulting in a slight angular deflection of the $3 \mathrm{D}$ point cloud in the direction of $Z$-axis. This causes the attitude error of the point cloud $C_{O}$. This error is denoted as $E_{\text {atti }}$, and we quantify $E_{\text {atti }}$ by Formula (11)

$$
E_{\text {atti }}=\left|\boldsymbol{n}_{1} \cdot \boldsymbol{i}\right|+\left|\boldsymbol{n}_{3} \cdot \boldsymbol{i}\right|+\left|\boldsymbol{n}_{2} \cdot \boldsymbol{j}\right|+\left|\boldsymbol{n}_{4} \cdot \boldsymbol{j}\right|
$$

In Formula (11), we quantify the attitude error $E_{\text {atti }}$ of the point cloud $C_{O}$ through the sum of the absolute values of the inner product of the unit vector. The vectors $\boldsymbol{n}_{1}, \boldsymbol{n}_{2}, \boldsymbol{n}_{3}$, $n_{4}$ are unit normal vectors extracted from the point cloud $C_{O}$, and they correspond to the 4 walls shown in Figure 7. The vectors $i$ and $j$ are unit vectors of the $X$-axis and $Y$-axis of the coordinate frame $O$, respectively. The vectors $i$ and $j$ are fixed because the coordinate frame $O$ is fixed relative to the stationary part of the prototype, the positive direction of $X$-axis parallel to the front direction of the prototype (see Section 3.2). In Figure 7, we put the prototype in the conference room in a fixed and not-skewed way. Therefore, the coordinate frame $O$ is fixed and not-skewed relative to the conference room.

If there is not any skew for point cloud $C_{O}$ relative to coordinate frame $O$, the vectors $n_{1}$ and $n_{3}$ are perpendicular to the vector $\boldsymbol{i}$, and the vectors $\boldsymbol{n}_{2}$ and $\boldsymbol{n}_{4}$ are perpendicular 
to the vector $j$, as a result of which, $E_{\text {atti }}=0$. While the angular deflection of the $3 \mathrm{D}$ point cloud $C_{O}$ is between $-90^{\circ}$ and $+90^{\circ}, E_{\text {atti }}$ increases as the absolute value of the angular deflection increases. Obviously, in the actual situation, the angular deflection of the 3D point cloud $C_{O}$ cannot exceed the above range.

We denote the skew angle of the point cloud $C_{O}$ as $\varphi_{\text {error }}$, that is, the angular deviation of the point cloud $C_{O}$ relative to the correct attitude in the direction of $Z$-axis. If we rotate point cloud $C_{O}$ by angle $\varphi_{\text {error }}$ to make it coincide with the correct attitude, then we can make $E_{\text {atti }}=0$. Since the vectors $\boldsymbol{n}_{1}, \boldsymbol{n}_{2}, \boldsymbol{n}_{3}, \boldsymbol{n}_{4}$ are extracted from the point cloud $\boldsymbol{C}_{O}$, while we rotate point cloud $\boldsymbol{C}_{O}$, vectors $\boldsymbol{n}_{1}, \boldsymbol{n}_{2}, \boldsymbol{n}_{3}, \boldsymbol{n}_{4}$ are also be rotated. We substitute the rotated vectors into Formula (11), and we can get the following formula:

$$
f\left(\varphi_{\text {error }}\right)=\left|\boldsymbol{R}_{\text {error }} \boldsymbol{n}_{1} \cdot \boldsymbol{i}\right|+\left|\boldsymbol{R}_{\text {error }} \boldsymbol{n}_{3} \cdot \boldsymbol{i}\right|+\left|\boldsymbol{R}_{\text {error }} \boldsymbol{n}_{2} \cdot \boldsymbol{j}\right|+\left|\boldsymbol{R}_{\text {error }} \boldsymbol{n}_{4} \cdot \boldsymbol{j}\right|
$$

where $\boldsymbol{R}_{\text {error }}$ is a rotation matrix calculated according to angle $\varphi_{\text {error }}$

$$
\boldsymbol{R}_{\text {error }}=\left[\begin{array}{ccc}
c\left(\varphi_{\text {error }}\right) & -s\left(\varphi_{\text {error }}\right) & 0 \\
s\left(\varphi_{\text {error }}\right) & c\left(\varphi_{\text {error }}\right) & 0 \\
0 & 0 & 1
\end{array}\right]
$$

It is easy to find that, ideally:

$$
f\left(\varphi_{\text {error }}\right)=0
$$

The angle $\varphi_{\text {error }}$ is numerically equal to the difference between the actual value and the expected value of angle $\alpha$, that is:

$$
\varphi_{\text {error }}=\alpha-30^{\circ}
$$

where $30^{\circ}$ is the expected value of the angle $\alpha$. Combining Formulas (12), (13), (15), we can change the independent variable of the function $f\left(\varphi_{\text {error }}\right)$ from $\varphi_{\text {error }}$ to $\alpha$, that is, function $f_{\text {cost }}(\alpha)$ can be constituted, as shown below:

$$
f_{\text {cost }}(\alpha)=\left|\boldsymbol{R}_{\text {error }} \boldsymbol{n}_{1} \cdot \boldsymbol{i}\right|+\left|\boldsymbol{R}_{\text {error }} \boldsymbol{n}_{3} \cdot \boldsymbol{i}\right|+\left|\boldsymbol{R}_{\text {error }} \boldsymbol{n}_{2} \cdot \boldsymbol{j}\right|+\left|\boldsymbol{R}_{\text {error }} \boldsymbol{n}_{4} \cdot \boldsymbol{j}\right|
$$

where $\boldsymbol{R}_{\text {error }}$ has been changed to:

$$
\boldsymbol{R}_{\text {error }}=\left[\begin{array}{ccc}
c\left(\alpha-30^{\circ}\right) & -s\left(\alpha-30^{\circ}\right) & 0 \\
s\left(\alpha-30^{\circ}\right) & c\left(\alpha-30^{\circ}\right) & 0 \\
0 & 0 & 1
\end{array}\right]
$$

It is easy to find that, ideally:

$$
f_{\text {cost }}(\alpha)=0
$$

Next, we need to find the actual value of angle $\alpha$. This is an optimization problem, our goal is to find $\alpha^{*}$ through:

$$
\alpha^{*}=\operatorname{argmin} F_{\text {cost }}(\alpha)
$$

where

$$
F_{\text {cost }}(\alpha)=\frac{1}{2} \sum_{m=1}^{\mathrm{M}}\left(f_{\text {cost }}\left(\alpha_{m}\right)^{2}\right)
$$

In Formula (20), $\mathrm{M}$ is the total number of measurements, and $m$ is the serial number of a certain measurement. The optimization requires multiple 3D scans, because it is necessary to eliminate the interference of random factors, so that the actual value of angle $\alpha$ can be estimated more accurately.

We did 50 repeated scans in the highest resolution mode (mode 7, see Table 1) according to the method described in Figure 7, and used these $503 \mathrm{D}$ point clouds $C_{O}$ as the input of the optimization algorithm, that is, $\left\{C_{O}^{1}, C_{O}^{2}, C_{O}^{3}, \ldots, C_{O}^{50}\right\}$. The optimized value of angle 
$\alpha$ is the output of the algorithm. The reason why mode 7 is used is that in lower resolution mode (such as mode 1), the points in point cloud $C_{\text {tri }}$ are sparser, so that the error of the 3D coordinate of the center point $c$ is bigger. The inaccuracy of point $c$ leads to the inaccuracy of angle $\beta$. Since the value of the angle $\alpha$ is constant, through Equation (10) we can know that the angle $\varphi_{\text {offset }}$ inherits the inaccuracy of the angle $\beta$, which ultimately leads to the inaccuracy of the attitude of point cloud $C_{O}$, as shown in Equations (8) and (9).

Although the attitude of point cloud $C_{O}$ has been corrected, there are still residual errors remaining. One stems from the above-mentioned inaccuracy of point $c$, which is particularly obvious in low-resolution mode. The other stems from the constant error of the angle $\alpha$ caused by the installation error, which depends on the accuracy of the installation and has nothing to do with the scanning mode. In low-resolution mode, the former is the main factor. The phenomenon is that the attitude of point cloud $\boldsymbol{C}_{O}$ obtained from several $3 \mathrm{D}$ scans is slightly different. In the high-resolution mode, the latter is the main factor. The phenomenon is that the attitude of the point cloud $C_{O}$ obtained from several 3D scans is almost the same, but there is a constant difference between the attitude of point cloud $C_{O}$ and the correct attitude. Here we focus on the latter, and calibrate the installation error of the triangular plate. Therefore, we use the highest resolution mode to collect data as the input of our optimization algorithm, for the purpose of avoiding the above-mentioned first type of error as much as possible, that is, the error caused by low resolution.

In our optimization algorithm, we use the Levenberg-Marquardt method [32,33] to estimate the angle $\alpha$. Because the initial value and the final optimization result are very close (the installation error is generally small), the initial value is set as $\alpha_{0}=30^{\circ}$. Our optimization algorithm is shown in Algorithm A1, which is in Appendix C.

By Algorithm A1, we can estimate the value of angle $\alpha, \alpha=29.3598^{\circ}$. In Equation (10) for the calculation of $\varphi_{\text {offset, }}$, the value of angle $\alpha$ is replaced from the expected value of $30^{\circ}$ to the value estimated by Algorithm A1.

Until now, we can accurately calculate the angle $\varphi_{\text {offset }}$ between point cloud $C_{O^{\prime}}$ relative to coordinate frame $O^{\prime}$ and point cloud $C_{O}$ relative to coordinate frame $O$. Since point cloud $C_{O^{\prime}}$ and angle $\varphi_{\text {offset }}$ are both known, point cloud $C_{O}$ can be calculated according to Equations (8) and (9). It is accurate both in shape or attitude.

Summarizing our method, in Sections 2 and 3, we obtained the 3D point cloud $C_{O^{\prime}}$, which has an uncertain attitude error, but no shape error. In Section 4.1, we calibrated the uncertain attitude error of the point cloud $\boldsymbol{C}_{O^{\prime}}$ by using a triangular plate. In Section 4.2, we corrected the installation error of the triangular plate. Through the above steps, we can use our prototype to obtain a 3D point cloud with correct shape and attitude. We have calibrated the matching error between the $2 \mathrm{D}$ lidar and the motor at low cost. Unlike the existing methods, we do not need a sensor to measure the rotation angle of the motor shaft, which is costly.

\section{Experiments}

5.1. Effectiveness Test

\subsubsection{Data of Experiments}

According to the method described in Figure 7, a total of 70 point clouds were collected, which can be divided into 2 groups, one is without attitude correction, and the other is with attitude correction. In each group, 7 modes of the prototype were tested respectively, and 5 point clouds were collected in each mode.

First, we show the data qualitatively. In Figure 8, the top views of these 70 3D point clouds are listed, which can show the attitude of the 3D point cloud. The red 3D point cloud was obtained without attitude correction (that is, point cloud $C_{O^{\prime}}$ ), and the blue 3D point cloud was obtained with attitude correction (that is, point cloud $C_{O}$ ).

It can be found from Figure 8 that the attitudes of the 3D point clouds without correction (which are red) are uncertain. This uncertainty exists not only between different modes, but also between different 3D point clouds in the same mode. In comparison, the attitudes of the 3D point clouds with correction (which are blue) are roughly constant. In 
the following, the attitudes of 3D point clouds will be showed quantitatively by the curves of data.

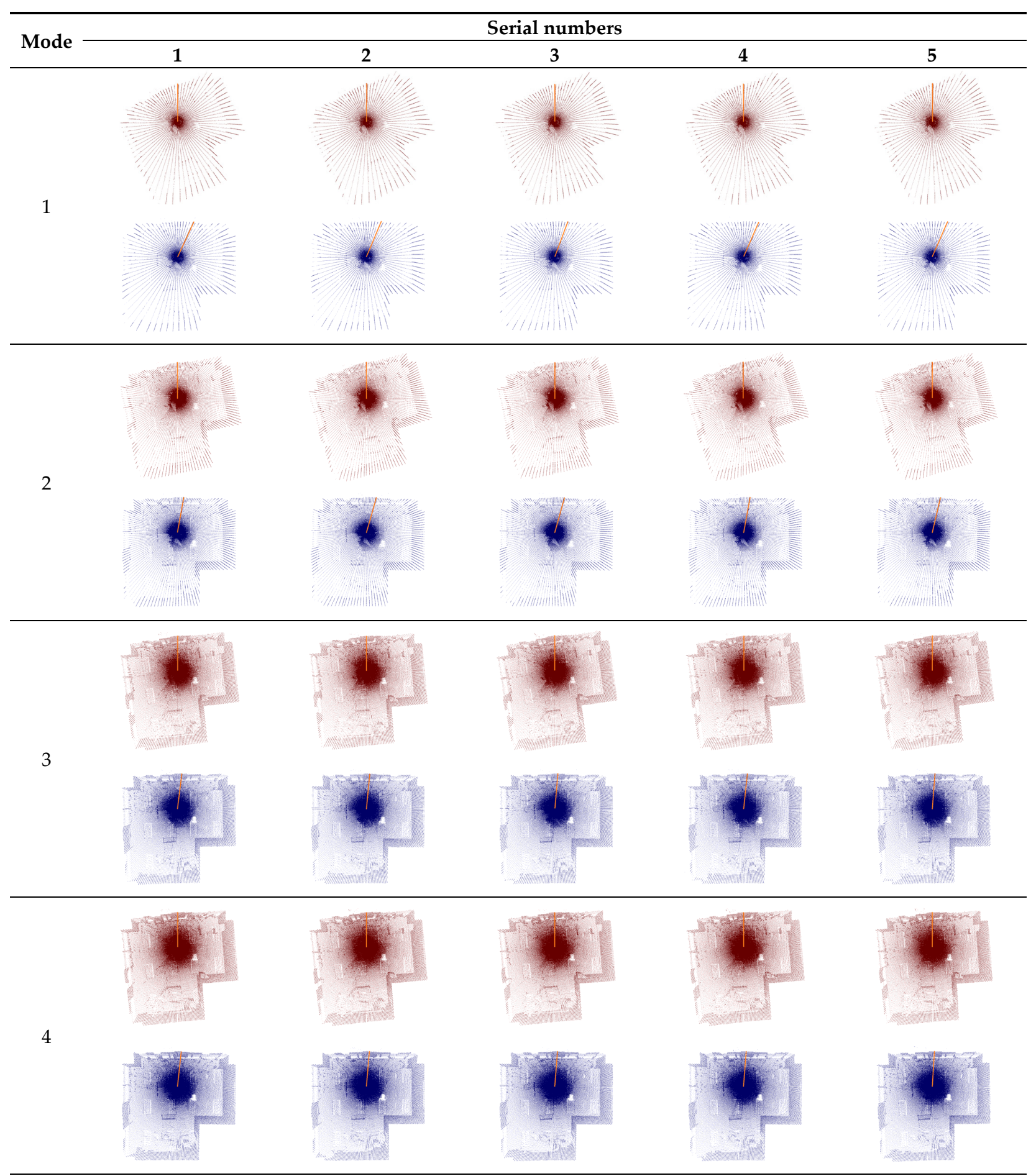

Figure 8. Cont. 


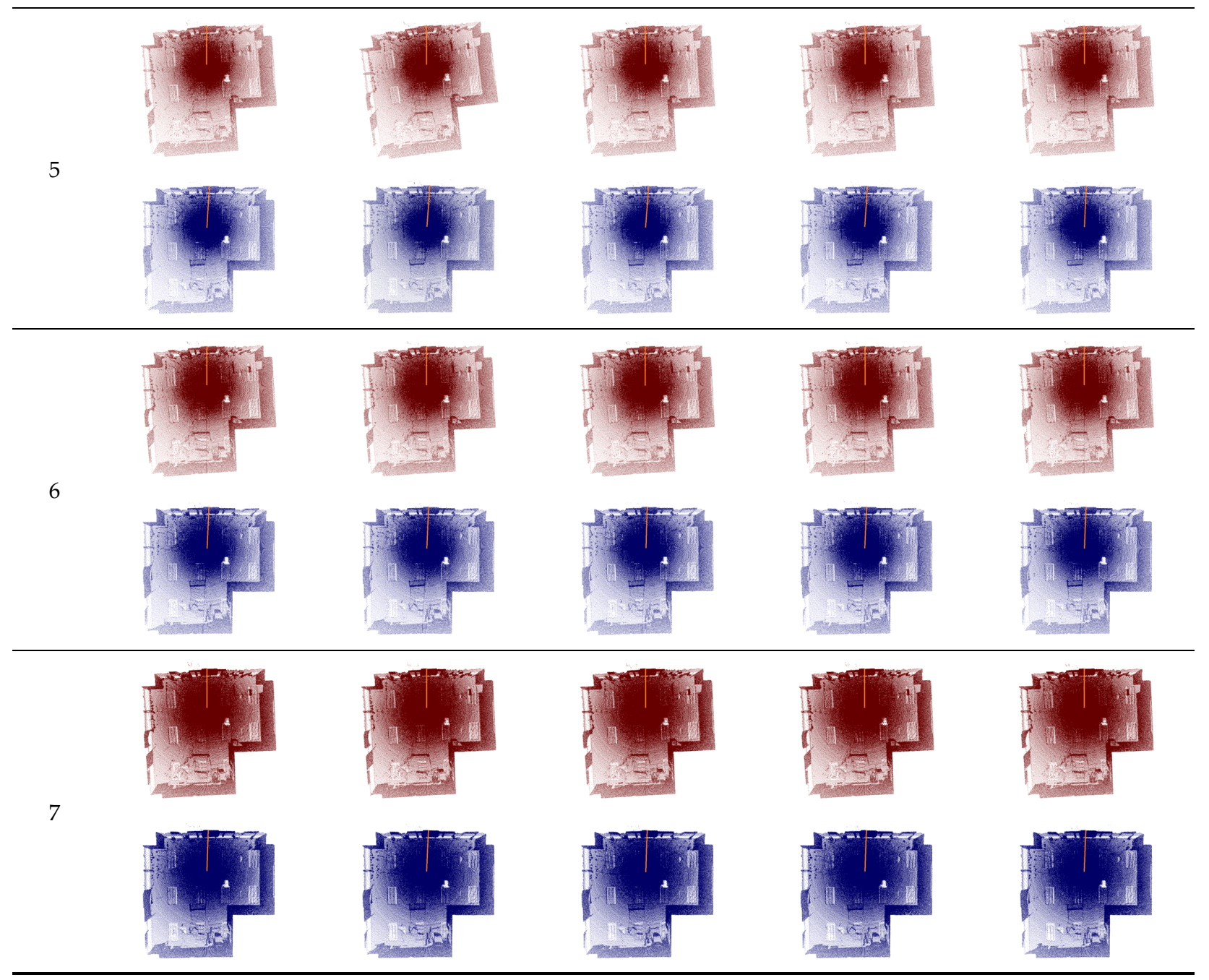

Figure 8. Top views of 70 3D point clouds.

In order to highlight the strip blank area and strip overlap area of each 3D point cloud, we marked them by a thick orange line. It is the position of the motor shaft when a 3D scanning starts. For the 3D point clouds with attitude correction, although their attitudes are roughly the same, the positions of the thick orange lines relative to the 3D point clouds are not the same. As mentioned above, the thick orange line shows the position of the motor shaft when a rotating 2D lidar starts a 3D scan, that is, the position of the $Y^{\prime}$-axis in the coordinate frame $O^{\prime}$. Since this position is not constant for each $3 \mathrm{D}$ scan, the position of thick orange line relative to the 3D point cloud is different for different 3D point clouds.

Next, we show the data quantitatively. We calculated the angle $\varphi_{\text {error }}$ of each 3D point cloud in Figure 8 through Equations (12)-(14). It is worth noting that $f\left(\varphi_{\text {error }}\right)=0$ in Formula (14) is under ideal conditions. During data processing, we calculated the $\varphi_{\text {error }}$ that minimizes $f\left(\varphi_{\text {error }}\right)$ as the result.

The values of angle $\varphi_{\text {error }}$ of 70 3D point clouds are shown in Figure 9. We used a line chart to make it more intuitive. The horizontal axis is the number of the mode, that is, mode 1 to mode 7 of the prototype. The vertical axis is the value of angle $\varphi_{\text {error }}$, its units are degrees. Looking down on a 3D point cloud, if the offset is clockwise relative to the correct attitude, $\varphi_{\text {error }}$ is positive, while a counterclockwise offset relative to the correct attitude, indicates negative error. The red line corresponds to the $3 \mathrm{D}$ point clouds without attitude correction, and the blue line corresponds to the 3D point clouds with attitude correction. For each line, it is divided into 7 segments by 7 modes, and there are 5 sampling points in each mode, which corresponding to $53 \mathrm{D}$ point clouds collected in this mode. 


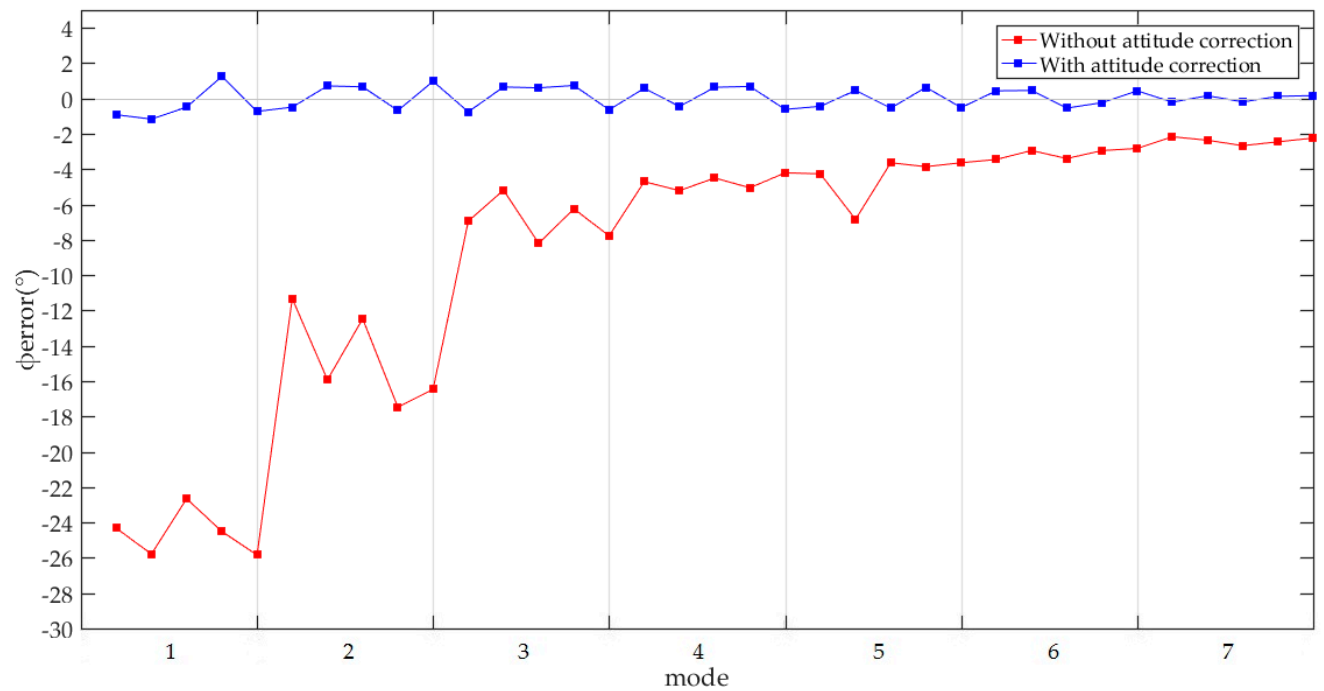

Figure 9. The values of angle $\varphi_{\text {error }}$ of $703 \mathrm{D}$ point clouds.

For the values of angle $\varphi_{\text {error }}$ of the 5 sampling points in each mode, we calculated the maximum, average and minimum values respectively, as shown in Figure 10.

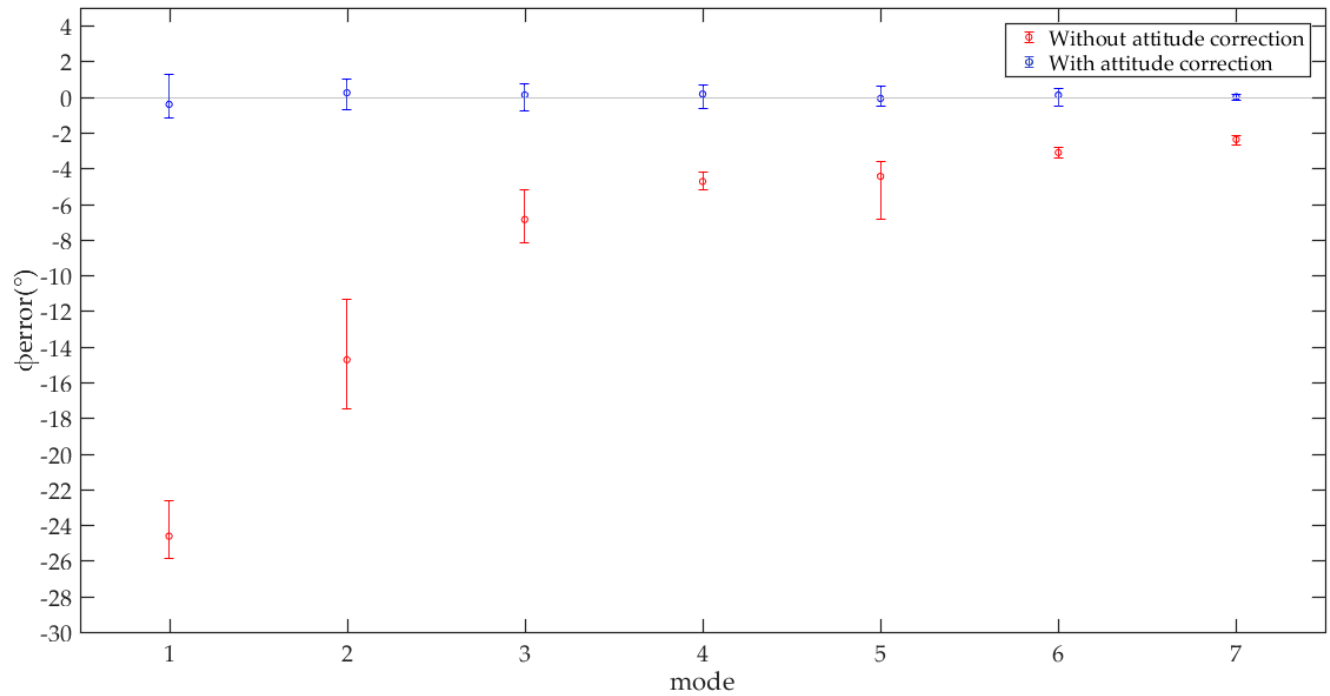

Figure 10. The maximum, average, and minimum values of angle perror of 5 sampling points in each mode.

Through the above experimental data, we can know the following points.

\subsubsection{Characteristics of Uncertain Attitude Error}

Here, we only considered the sample data without attitude correction. The characteristics of uncertain attitude error were found out.

From mode 1 to mode 7 , there is an obvious decreasing trend for the value of the uncertain attitude error. According to Section 2, an important reason for the uncertain attitude error of the 3D point cloud is the uncertain time deviation between the starting time of the 2D lidar and the starting time of the motor). Although the value of this time deviation is uncertain, it changes within a certain range. Therefore, in general, the faster the motor shaft rotates during this time deviation, the larger the attitude error of the 3D point cloud tends to be. The red part of Figures 8-10 verify this analysis. From mode 1 to mode 7 , the scanning resolution of a rotating 2D lidar is getting higher and higher, and the speed of the motor shaft is getting slower and slower. Therefore, there is a tendency that the attitude error is reduced. However, individual cases that violate this trend still 
exists. There is a sampling point in the segment corresponding to mode 5 of the red line in Figure 9 (the 22nd point), and its attitude error is much larger than other sampling points in the mode 5 segment, and also much larger than the sampling points in the mode 4 segment. During our tests, cases like this are not unique. This is because although there is a strong correlation between the uncertain attitude error of the 3D point cloud and the speed of the motor shaft, other factors also count. First, the value of the time deviation is uncertain. The response time of the photoelectric switch and the acceleration characteristics of the motor may also have impacts on the uncertain attitude error. We have analyzed this in detail in Section 2.

From mode 1 to mode 7 , there is an obvious decreasing trend for the uncertainty of the uncertain attitude error. During the uncertain time deviations, the faster the shaft of the motor rotates, the greater the uncertainty of the time deviation will be transformed into the uncertainty of the attitude of the 3D point cloud. The red part of Figure 10 validates this analysis, which shows the maximum, average, and minimum attitude errors of the 5 3D point cloud collected in each mode. Obviously, there is an overall trend that the higher the resolution of the scanning mode, the closer these three values are, which means that the uncertainty of the uncertain attitude error turns smaller.

\subsubsection{Characteristics of Our Algorithm}

Here, we only considered the sample data with attitude correction. The characteristics of our algorithm were found out.

It can be seen from the blue part of Figures 9 and 10 that for the high-resolution mode, the attitude error of the corrected 3D point cloud is smaller and more certain. For the low-resolution mode, the attitude error of the corrected 3D point cloud is larger and more uncertain. The reason has been analyzed in Section 4.2 , because the points in point cloud $C_{\text {tri }}$ obtained in the low-resolution mode are relatively sparse, so that the calculated center point $c$ has a large error. The error is shown in two points, one is that the calculated value of center point $c$ has a large deviation from the true value, the other is that the calculated value of center point $c$ is greatly uncertain in each 3D scan. The above two points will lead to a large and uncertain error of angle $\beta$, because the angle $\beta$ is calculated according to the 3D coordinates of the point $c$. As a result, a large and uncertain error of angle $\varphi_{\text {offset }}$ is caused. This can be seen from Formula (10). Where $\alpha$ is a constant value. According to Formulas (8) and (9), this can finally result in a large and uncertain attitude error of the point cloud $C_{O}$.

\subsubsection{Effectiveness of Our Algorithm}

Here, we compared the sample data without attitude correction and the sample data with attitude correction to evaluate the effectiveness of our algorithm.

It can be seen from the value of $\varphi_{\text {error }}$ that the attitude error is eliminated by our algorithm. This can be clearly seen from Figures $8-10$, the 3D point clouds without attitude correction (which is red) are obviously skew, and there are an obvious deviations between their $\varphi_{\text {error }}$ and 0 , while the attitudes of the 3D point clouds with attitude correction (which is blue) are correct, and their $\varphi_{\text {error }}$ approach 0 .

It can be seen from the uncertainty of $\varphi_{\text {error }}$ that, in general, in the same mode, the uncertainty of $\varphi_{\text {error }}$ of a 3D point cloud with attitude correction is smaller than that of a 3D point cloud without attitude correction (as shown in Figure 10). Comparing the red part and blue part of Figure 10, our algorithm reduces the uncertainty of $\varphi_{\text {error }}$, especially in low-resolution mode. In high-resolution mode, our algorithm does not show obvious performance in reducing the uncertainty of $\varphi_{\text {error }}$. After all, in high-resolution mode, the uncertainty of the attitude of the 3D point cloud is very small-even without attitude correction. However, mutation point which is similar to the 22 nd point of the red line showed in Figure 9 cannot be excluded. Our algorithm can deal with this situation. 
Our algorithm is effective, whether it is to reduce the attitude error of the 3D point cloud, or to reduce the uncertainty of the 3D point cloud attitude. As for whether the accuracy of our algorithm can meet the application requirements, we will analyze it below.

\subsection{Accuracy Test and Application Evaluation}

In this section, we evaluated whether the accuracy of our method can meet the requirements of the application. We used the 3D mapping of indoor autonomous mobile robots as the actual application, because a rotating $2 \mathrm{D}$ lidar is widely used in the $3 \mathrm{D}$ mapping of indoor mobile robots [17,34-36], and the 2D lidar Hokuyo UST-10LX [30] used in our prototype is a product mainly used for indoor. In this application, the main negative effect of the attitude error of the $3 \mathrm{D}$ point cloud is to make the robot navigate in the wrong direction, thereby increasing the risk of crashing the wall. The variation curve of the mapping error $E_{\text {map }}$ with distance $d$ in 7 modes is shown in Figure 11. The mapping error $E_{\text {map }}$ is calculated by the following formula

$$
E_{\text {map }}=d \cdot \tan \left(\varphi_{\text {error }}\right)
$$
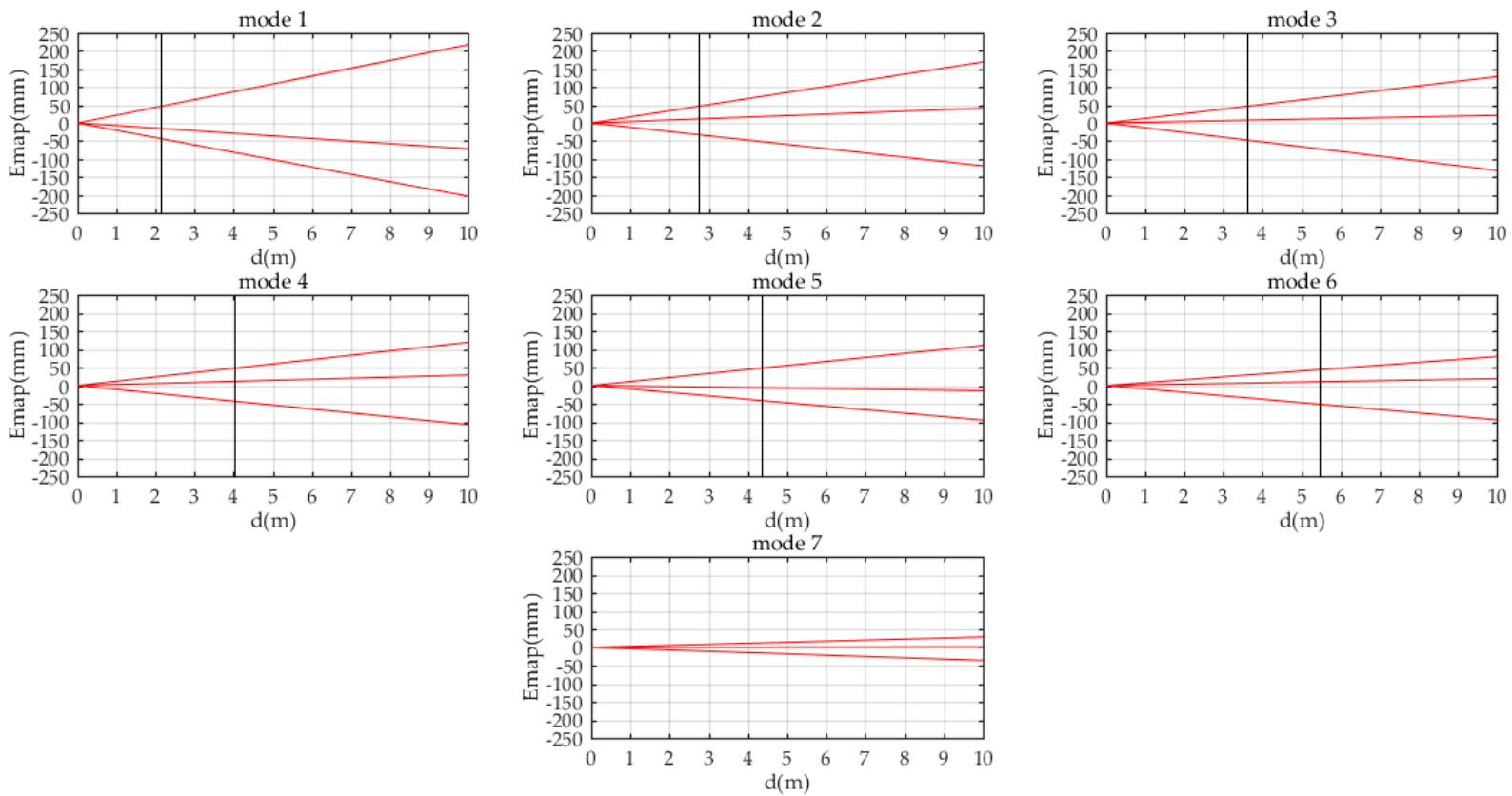

Figure 11. Variation curve of mapping error with distance in 7 modes.

There are 7 graphs in Figure 11, which correspond to the 7 scan modes of the prototype. The horizontal axis of the graph is the distance $d$, its units are meters. The vertical axis of the graph is the mapping error $E_{\mathrm{map}}$, its units are millimeters. The maximum range of the horizontal axis $d$ is $10 \mathrm{~m}$, which is the range of the 2D lidar we used in our prototype. The three curves in each graph are the variation curve of $E_{\text {map }}$ with distance $d$ calculated according to the maximum, average and minimum values of angle $\varphi_{\text {error }}$, respectively. The area between the top curve and the bottom curve represents the variation range of the attitude error of the 3D point cloud in this mode.

We took $\pm 50 \mathrm{~mm}$ as a standard, and marked the corresponding $d$ while the mapping error $E_{\text {map }}$ of each mode is limited within $\pm 50 \mathrm{~mm}$ in Figure 11. Even in mode 1, which has the lowest accuracy, the mapping error $E_{\text {map }}$ can be limited within $\pm 50 \mathrm{~mm}$ at $d=2.2996 \mathrm{~m}$. In mode 7 , the mapping error $E_{\text {map }}$ at the measuring range $d=10 \mathrm{~m}$ can be limited within $\pm 50 \mathrm{~mm}$. For 3D mapping of indoor autonomous mobile robots, a mapping error of $\pm 50 \mathrm{~mm}$ is acceptable. After all, the accuracy of the 2D lidar Hokuyo UST-10LX used in our prototype is $\pm 40 \mathrm{~mm}$ [30]. Moreover, after the robot has approached a target (such 
as a door), the rotating $2 \mathrm{D}$ lidar mounted on it can 3D scan again to refresh the 3D map. According to Formula (21), the mapping error $E_{\text {map }}$ of this target will be reduced because the distance is closer.

The above analysis has verified that the accuracy of our method can meet the requirements of 3D mapping of indoor autonomous mobile robots.

\section{Conclusions}

For a rotating 2D lidar, the inaccurate matching between the 2D lidar and the motor is an important cause of the shape and attitude error of the 3D point cloud. To solve this problem, existing methods need to synchronize the 2D lidar data and the motor shaft by measuring the angle position of the motor shaft. To measure the angle position of the motor shaft, either a complicated and expensive servo system with absolute positioning function is required, or the motor needs to be equipped with a precise angular displacement sensor, such as an absolute encoder. This will greatly increase the cost.

Our method eliminates the shape and attitude error of the 3D point cloud caused by the inaccurate matching between the 2D lidar and the motor, without using a servo system or encoder. First, we modified the sequence between the motor and the 2D lidar to make the motor start working earlier and stop working later than the 2D lidar, so that the shape of the 3D point cloud is no longer affected by the acceleration and deceleration of the motor, a 3D point cloud with correct shape can be obtained. Next, we eliminated the uncertain error of the attitude of the 3D point cloud through a triangular plate fixed on the prototype. Finally, we calibrated the installation error of the triangular plate by using Levenberg-Marquardt method. We verified the effectiveness and accuracy of our method by experiments. The results show that the collected 3D point cloud can meet the requirements of 3D mapping of an indoor autonomous mobile robot. Through our work, while reducing the cost, the accuracy is ensured. Our work provides a new idea for the cost reduction of a rotating $2 \mathrm{D}$ lidar.

Our method can not only calibrate the matching error between the 2D lidar and the motor, but also partially calibrate the installation error between the 2D lidar and the motor shaft. The installation error is 6-DOF, containing a 3-DOF translation and 3-DOF rotation [4]. The direction of one of the rotation errors is the same as the rotation direction of the motor shaft. It will not cause the shape error of the 3D point cloud, but it will cause the attitude error of the 3D point cloud. Due to its characteristic that it will not cause the distortion in shape of the 3D point cloud, it is difficult to calibrate it [1,5], while our method can calibrate it.

As for future work, we have noticed that the real-time performance of a rotating $2 \mathrm{D}$ lidar is very poor, and its 3D scanning frequency is often not higher than $1 \mathrm{~Hz}$. Therefore, when a rotating $2 \mathrm{D}$ lidar is scanning, the movement of the platform carrying the rotating 2D lidar will cause the distortion of the 3D point cloud $[24,25,27]$. Therefore, for a rotating 2D lidar, it is not enough to obtain an accurate 3D point cloud in static state, it is necessary to study how to eliminate the distortion of the 3D point cloud in motion state. Our followup plan is to combine our method with an algorithm that calibrates the distortion of the $3 \mathrm{D}$ point cloud in a dynamic environment. We will try to collect an accurate 3D point cloud (which is accurate both in shape and attitude) in a dynamic environment, by using a low-cost rotating 2D lidar which does not require an angular sensor to measure the angle of motor shaft.

Author Contributions: Conceptualization, C.Y. and S.B.; methodology, C.Y.; software, C.Y., J.C., W.W., and D.Y.; validation, C.Y., J.C., W.W. and D.Y.; formal analysis, C.Y.; investigation, C.Y.; resources, S.B.; data curation, C.Y.; writing—original draft preparation, C.Y.; writing—review and editing, S.B., W.W., J.C. and D.Y.; visualization, C.Y.; supervision, S.B.; project administration, C.Y.; and funding acquisition, S.B. All authors have read and agreed to the published version of the manuscript.

Funding: This research was funded by Scientific and Technological Project of Hunan Province on Strategic Emerging Industry under grant number 2016GK4007, Beijing Natural Science Foundation 
under grant number 3182019, and National Natural Science Foundation of China under grant number 91748101.

Acknowledgments: We thank Yanan Wang for technical support.

Conflicts of Interest: The authors declare no conflict of interest.

\section{Appendix A}

The notations used throughout this paper have been listed in Table A1.

Table A1. Nomenclature.

\begin{tabular}{|c|c|}
\hline Symbol & Explanation \\
\hline$\gamma$ & Angular resolution of rotating $2 \mathrm{D}$ lidar in the direction of motor shaft. \\
\hline$n$ & Number of 2D lidar scans included in a 3D scan. \\
\hline$f$ & The scanning frequency of 2D lidar is $f$, which can be found in its product manual. \\
\hline$T$ & The time used by a $3 \mathrm{D}$ scanning of a rotating $2 \mathrm{D}$ lidar is $T$. \\
\hline$\omega$ & The Angular velocity of the motor shaft in a rotating 2D lidar is $\omega$. \\
\hline$L$ & The coordinate frame of 2D lidar. \\
\hline$O^{\prime}$ & The coordinate frame of rotating unit (before attitude correction). \\
\hline$O$ & The coordinate frame of rotating unit (after attitude correction). \\
\hline$r$ & Ranging data of 2D lidar. \\
\hline$\theta$ & The azimuth angle in the scanning sector of the 2D lidar corresponding to the ranging data $r$. \\
\hline$p_{L}$ & A 3D point with respect to coordinate frame $L$, it is calculated according to the data of 2D LIDAR. \\
\hline $\boldsymbol{R}_{L}^{O^{\prime}}$ & Rotation matrix from coordinate frame $L$ to coordinate frame $O^{\prime}$ at the moment when a 3D scan of a rotating $2 \mathrm{D}$ lidar is started. \\
\hline$t_{L}^{O^{\prime}}$ & $\begin{array}{l}\text { The translation vector from coordinate frame } L \text { to coordinate frame } O^{\prime} \text { at the moment when a 3D scan of a rotating } 2 \mathrm{D} \text { lidar } \\
\text { is started. }\end{array}$ \\
\hline$\varphi_{\text {motor }}$ & Rotation angle of the motor shaft corresponding to the ranging data $r$. \\
\hline$R_{\mathrm{M}}$ & The rotation matrix calculated according to the rotation angle $\varphi_{\text {motor }}$ of the motor shaft. \\
\hline $\mathrm{K}$ & The total number of points in the 3D point cloud obtained by a 3D scan of a rotating 2D lidar. \\
\hline$k$ & The ordinal number of a point in all points of a 3D point cloud. \\
\hline$p_{O^{\prime}}$ & A 3D point with respect to coordinate frame $O^{\prime}$, which is converted from point $p_{L}$ in coordinate frame $L$. \\
\hline$\varphi_{\text {offset }}$ & The deflection angle of the coordinate frame $O^{\prime}$ relative to the coordinate frame $O$ in the $Z$-axis direction. \\
\hline $\boldsymbol{R}_{O^{\prime}}^{O}$ & The rotation matrix calculated according to $\varphi_{\text {offset }}$. \\
\hline$p_{O}$ & A 3D point with respect to coordinate frame $O$, which is converted from point $p_{O^{\prime}}$ in coordinate frame $O^{\prime}$. \\
\hline$C_{O^{\prime}}$ & A 3D point cloud with respect to coordinate frame $\boldsymbol{O}^{\prime}$, which is collected by a rotating 2D lidar. \\
\hline$C_{\text {tri }}$ & A point cloud corresponding to the triangular plate extracted from the point cloud $C_{O^{\prime}}$. \\
\hline$c$ & The center point of the triangular plate. \\
\hline$\alpha$ & $\begin{array}{l}\text { The angle between the middle line of the triangular plate and the front direction of the prototype (which is also the positive } \\
\text { direction of the axis } X \text { of coordinate frame } O \text { ). }\end{array}$ \\
\hline$\beta$ & The angle between the middle line of the triangular plate and the axis $X^{\prime}$ of the coordinate frame $O^{\prime}$. \\
\hline$c_{O}$ & A 3D point cloud with respect to coordinate frame $O$, which is converted from point cloud $C_{O^{\prime}}$ through rigid body transformation. \\
\hline$n_{1} \sim n_{4}$ & The unit normal vectors of the planes corresponding to the 4 walls of the room in the point cloud $C_{O}$, respectively. \\
\hline$i, j$ & The unit normal vectors corresponding to $X$-axis and $Y$-axis of coordinate frame $O$, respectively. \\
\hline$E_{\text {atti }}$ & The attitude error of point cloud $C_{O}$, which is expressed as the sum of absolute values of vector inner product. \\
\hline$\varphi_{\text {error }}$ & The attitude error of point cloud $C_{O}$, which is expressed as an angle. \\
\hline$R_{\text {error }}$ & The rotation matrix calculated according to the angle $\varphi_{\text {error }}$. \\
\hline$f\left(\varphi_{\text {error }}\right)$ & A function showing the attitude error of the point cloud $\boldsymbol{C}_{O}$, with $\varphi_{\text {error }}$ as the independent variable. \\
\hline$f_{\text {cost }}(\alpha)$ & A function showing the attitude error of the point cloud $C_{O}$, with $\alpha$ as the independent variable. \\
\hline & The total number of 3D scans used for the calibration of the installation error of the triangular plate. \\
\hline$m$ & The serial number of a 3D scan while calibrating the installation error of the triangular plate. \\
\hline$F_{\text {cost }}(\alpha)$ & The total attitude error of point cloud $C_{O}$, which is expressed by least square method. \\
\hline $\mathbf{N}_{1} \sim \mathbf{N}_{4}$ & Data sets, formed by $\boldsymbol{n}_{1} \sim \boldsymbol{n}_{4}$ from 50 point clouds $C_{O}$, respectively. \\
\hline$k_{\text {itera }}$ & Parameter of Algorithm A1, the serial number of a iteration. \\
\hline$k_{\max }$ & Parameter of Algorithm A1, the maximum times of iteration. \\
\hline$\alpha_{0}$ & Parameter of Algorithm A1, the initial value of $\alpha$. \\
\hline$\varepsilon_{1}$ & Parameter of Algorithm A1, the first stopping criteria of the algorithm. \\
\hline$\varepsilon_{2}$ & Parameter of Algorithm A1, the second stopping criteria of the algorithm. \\
\hline$J(\alpha)$ & Parameter of Algorithm A1, Jacobian matrix. \\
\hline A & Parameter of Algorithm A1, $A=J(\alpha)^{\mathrm{T}} J(\alpha)$ \\
\hline$g$ & Parameter of Algorithm A1, $g=J(\alpha)^{\mathrm{T}} F_{\text {cost }}(\alpha)$ \\
\hline$\mu$ & Parameter of Algorithm A1, the damping parameter. \\
\hline$\tau$ & Parameter of Algorithm A1, a coefficient which is used to determine the initial value of $\mu$. \\
\hline$v$ & Parameter of Algorithm A1, a coefficient which is used to adjust the value of $\mu$ in each iteration. \\
\hline$a_{i i}$ & Parameter of Algorithm A1, the element in $A$. \\
\hline I & Parameter of Algorithm A1, unit matrix. \\
\hline$\Delta \alpha$ & Parameter of Algorithm A1, the gain of $\alpha$ in each iteration. \\
\hline$\rho$ & Parameter of Algorithm A1, gain ratio. \\
\hline & The distance from the object to a rotating 2D lidar. \\
\hline$E_{\text {map }}$ & The error of the 3D map at distance $d$, the map is obtained by a rotating 2D lidar which is calibrated by our method. \\
\hline
\end{tabular}




\section{Appendix B}

We referred to the method in $[7,10]$ to evaluate the accuracy of the shape of the 3D point cloud obtained by our prototype. The flatness of 4 surfaces (as shown in Figure A1) in the $3 \mathrm{D}$ point cloud were analyzed to evaluate the accuracy of the 3D point cloud in shape more comprehensively. We extracted 4 ideal planes from the 4 surfaces respectively and calculated the deviations from the points to the ideal planes. When selecting the surfaces, we carefully avoided the door and the projection screen hanging on the wall, because they might reduce the accuracy of the extraction of the planes. These 4 surfaces are named surface 1 to surface 4 , respectively.

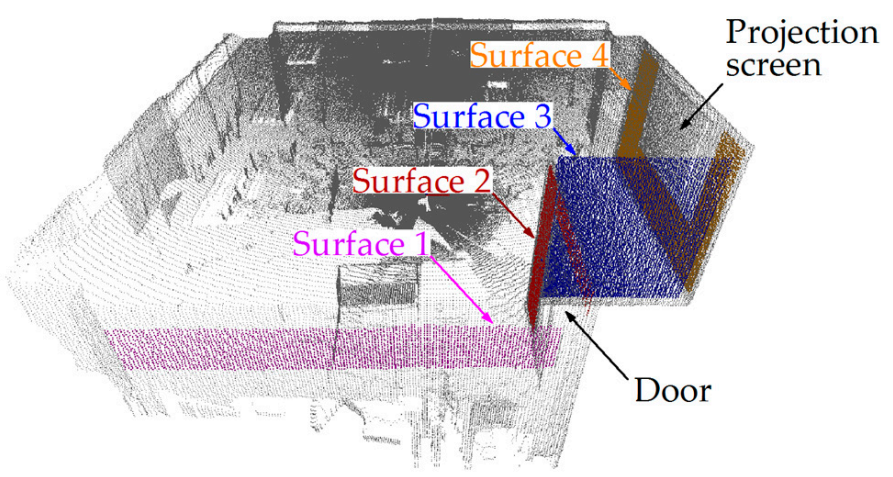

Figure A1. Four surfaces which were used to evaluate the accuracy of the 3D point cloud in shape. We marked them with different colors to distinguish them. The door and the projection screen were both avoided for they might reduce the accuracy of the extraction of the planes.

We evaluated the shape accuracy of the 3D point clouds obtained by our prototype in 7 modes, as shown in Figure A2. In Figure A2, the 3D view, side views $(X-Z$ view and $\mathrm{Y}-\mathrm{Z}$ view) of each surface are included, as well as the distribution of the deviations from each point to the ideal plane. The horizontal axis of the distribution graph describes the signed value of the deviations, while the vertical axis describes the number of points corresponding to each deviation, and the total number of the points is equal to the number of points contained in this surface.

From Figure A2, the following conclusions can be drawn.

(a) We can observe that the points in each surface are roughly evenly distributed on both sides of the ideal plane, which indicates that the surface and the ideal plane fit well. This shows that the shapes of these surfaces are very close to planes. Since they were obtained by scanning to the walls using our prototype, this can further indicate that the shape of the $3 \mathrm{D}$ point cloud collected by our prototype is correct.

(b) For each surface, its $\mathrm{X}-\mathrm{Z}$ view and $\mathrm{Y}-\mathrm{Z}$ view show that the distribution range of the points which are distributed at both sides of the ideal plane is roughly coincident with the accuracy of the 2D lidar (which is $\pm 40 \mathrm{~mm}$ ) used in our prototype. There is no case where a large number of points exceed this range, which indicates that the surface is obviously distorted.

(c) From the $\mathrm{X}-\mathrm{Z}$ view and $\mathrm{Y}-\mathrm{Z}$ view of each surface in Figure A2, we can find that very few sampling points exceed the range of $\pm 0.04 \mathrm{~m}$, which is the accuracy of the $2 \mathrm{D}$ lidar. So we can know that the shape error of the 3D point cloud caused by the self-reasons of our prototype (such as mechanical error, etc.) is relatively small compared to the error 2D lidar. Under the influence of the latter, the former is almost hardly noticeable.

(d) From the distribution graphs of the deviations, we can find a law of normal distribution (for surfaces with more points, their normal distributions are more obvious), which indicates that the deviation values from the points to the ideal plane are approximately normally distributed. This verifies the previous conclusion, that is, the shape of the 3D point cloud collected by our prototype is correct. If the 3D point cloud is distorted, the distribution of the deviations from the points to the ideal plane will 
be severely affected by the shape of the distorted surface, and it will be not easy to find out a normal distribution in the distribution graphs of the deviations.

Since the shape accuracy of the 3D point cloud has been verified, we can focus on the calibration of the attitude error of the 3D point cloud.

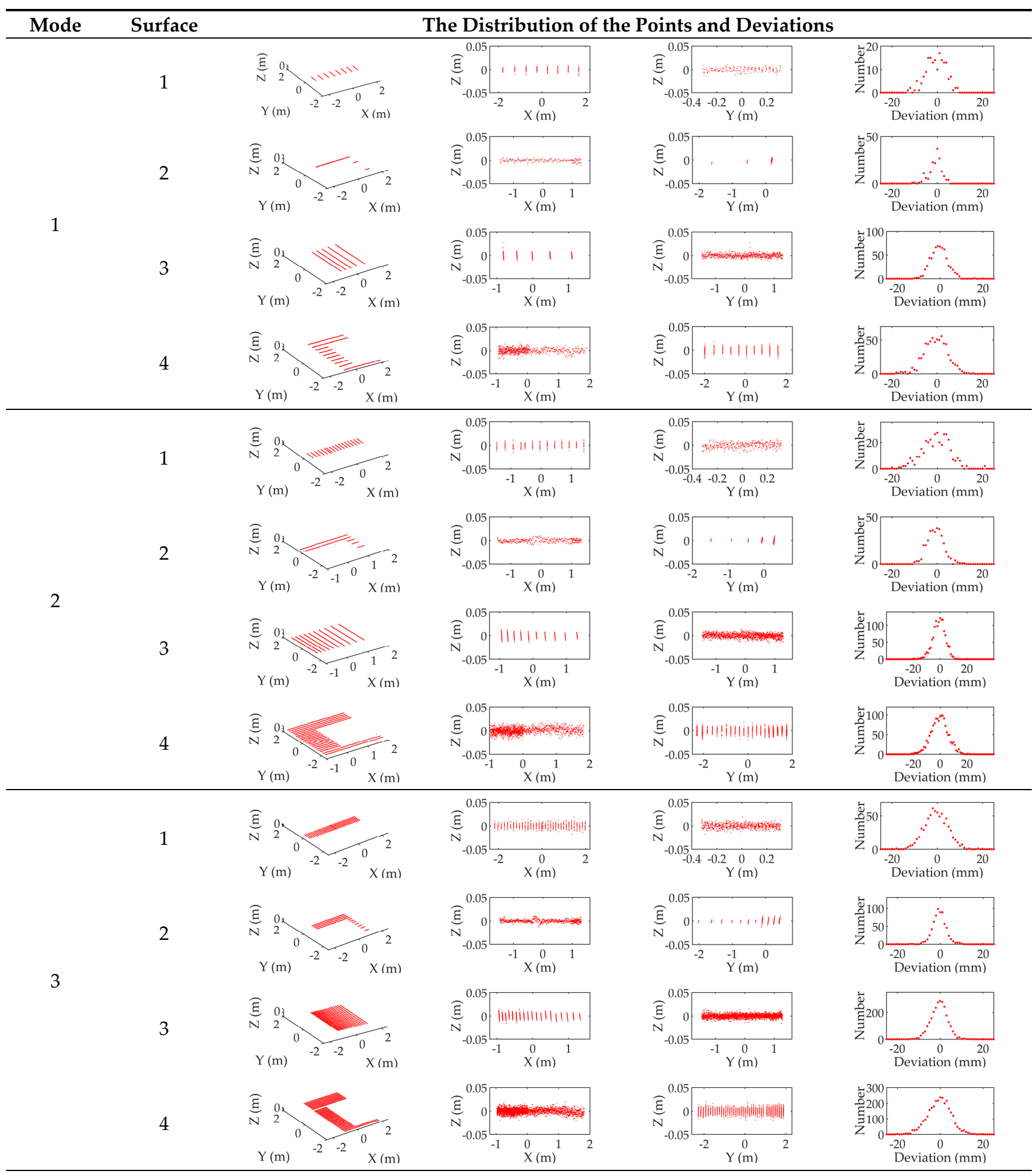

Figure A2. Cont. 


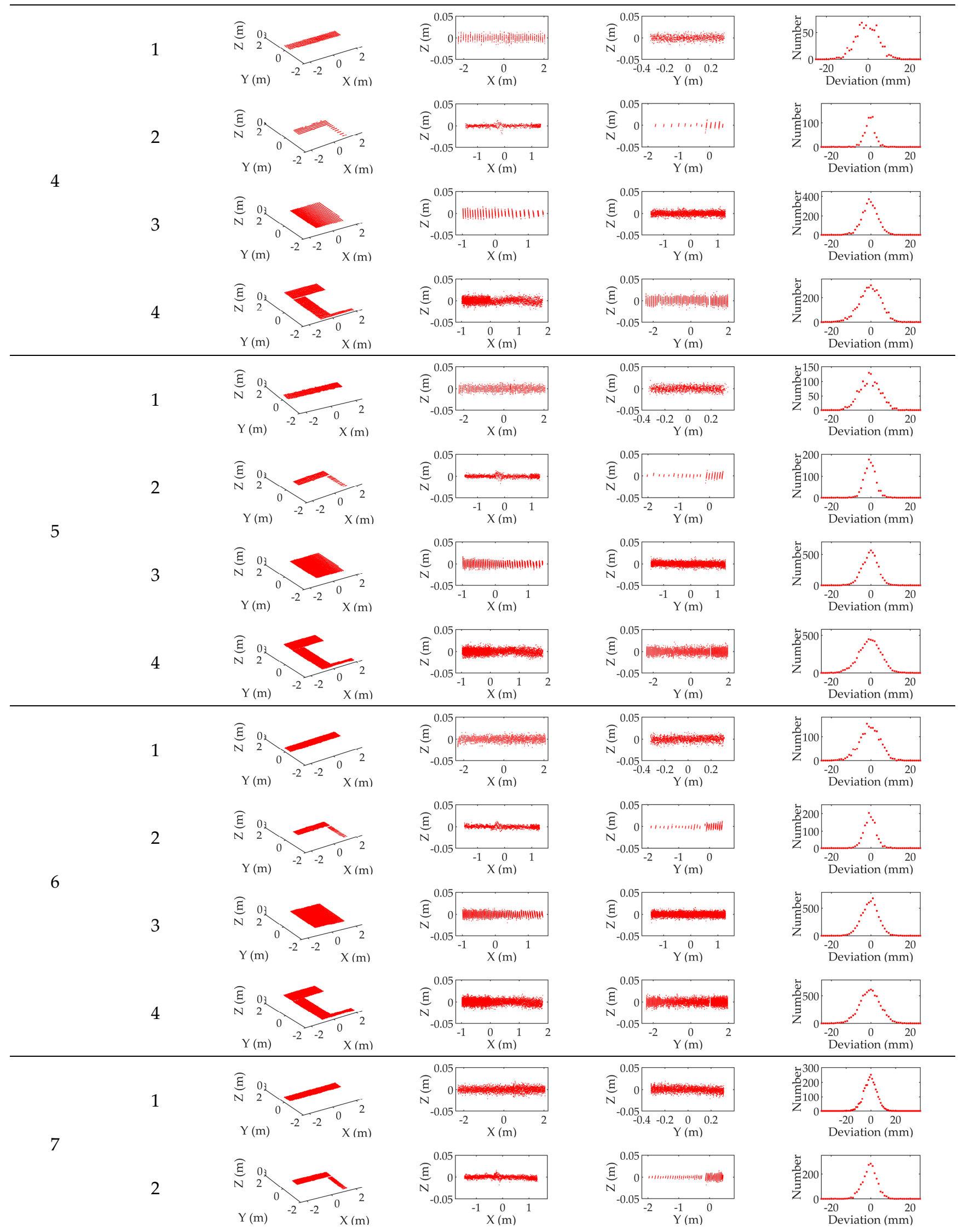

Figure A2. Cont. 


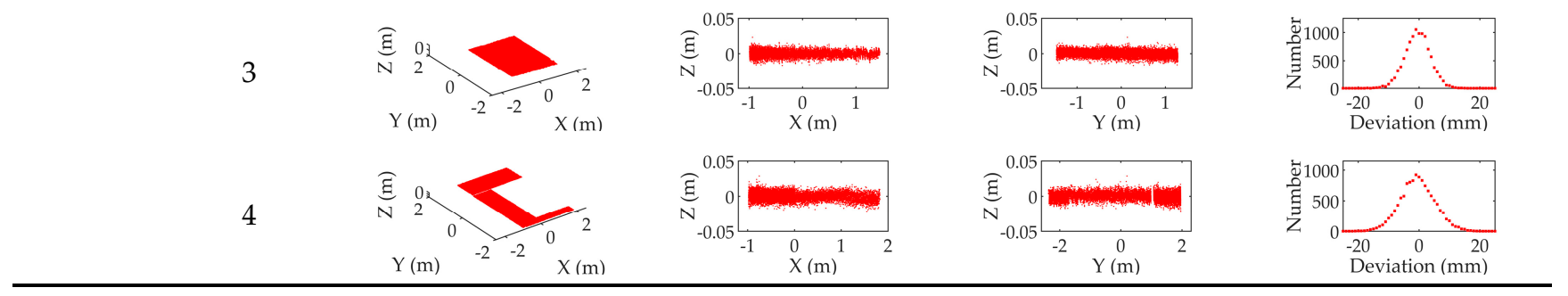

Figure A2. The evaluation of the shape accuracy of the 3D point clouds obtained by our prototype in 7 modes.

\section{Appendix C}

Our algorithm is shown in Algorithm A1.

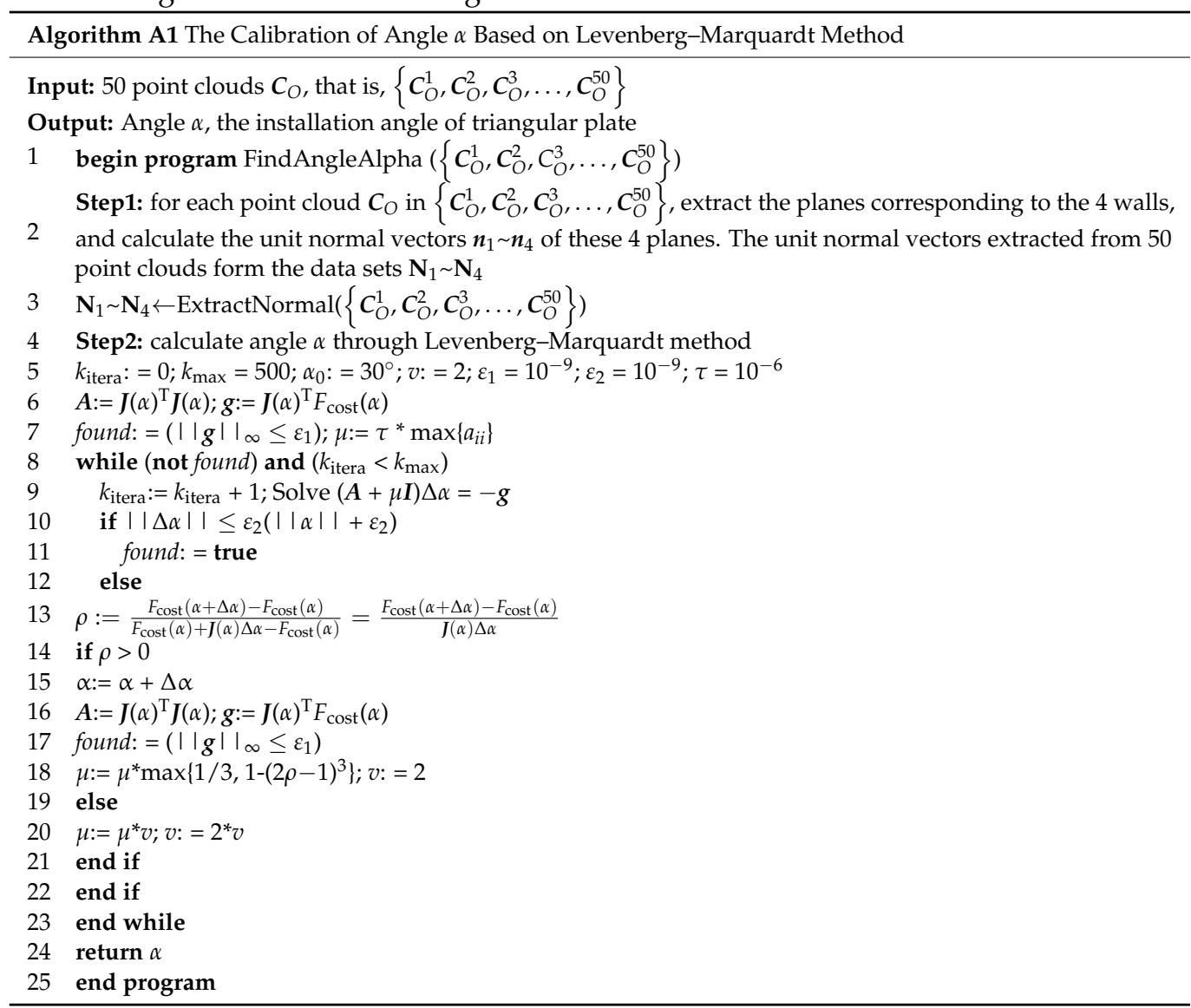

\section{References}

1. Morales, J.; Martinez, J.L.; Mandow, A.; Reina, A.J.; Pequenoboter, A.; Garciacerezo, A. Boresight Calibration of Construction Misalignments for 3D Scanners Built with a 2D Laser Rangefinder Rotating on Its Optical Center. Sensors 2014, 14, 20025-20040. [CrossRef] [PubMed]

2. Morales, J.; Martinez, J.L.; Mandow, A.; Pequenoboter, A.; Garciacerezo, A. Design and development of a fast and precise low-cost 3D laser rangefinder. In Proceedings of the International Conference on Mechatronics, Istanbul, Turkey, 13-15 April 2011; pp. 621-626.

3. Wulf, O.; Wagner, B. Fast 3d scanning methods for laser measurement systems. In International Conference on Control Systems and Computer Science; Editura Politehnica Press, 2003. Available online: https://www.researchgate.net/publication/228586709_Fast_ 3D_scanning_methods_for_laser_measurement_systems (accessed on 6 January 2021).

4. Kang, J.; Doh, N.L. Full-DOF Calibration of a Rotating 2-D LIDAR with a Simple Plane Measurement. IEEE Trans. Robot. 2016, 32, 1245-1263. [CrossRef] 
5. Gao, Z.; Huang, J.; Yang, X.; An, P. Calibration of rotating 2D LIDAR based on simple plane measurement. Sens. Rev. 2019, 39, 190-198. [CrossRef]

6. Alismail, H.; Browning, B. Automatic Calibration of Spinning Actuated Lidar Internal Parameters. J. Field Robot. 2015, 32, 723-747. [CrossRef]

7. Zeng, Y.; Yu, H.; Dai, H.; Song, S.; Lin, M.; Sun, B.; Jiang, W.; Meng, M.Q.H. An Improved Calibration Method for a Rotating 2D LIDAR System. Sensors 2018, 18, 497. [CrossRef] [PubMed]

8. Martinez, J.L.; Morales, J.; Reina, A.J.; Mandow, A.; Pequeno-Boter, A.; Garcia-Cerezo, A. Construction and Calibration of a Low-Cost 3D Laser Scanner with 360 degrees Field of View for Mobile Robots. In Proceedings of the 2015 IEEE International Conference on Industrial Technology (ICIT), Seville, Spain, 17-19 March 2015; pp. 149-154.

9. Murcia, H.F.; Monroy, M.F.; Mora, L.F. 3D Scene Reconstruction Based on a 2D Moving LiDAR. In International Conference on Applied Informatics; Springer: Cham, Switzerland, 2018.

10. Olivka, P.; Krumnikl, M.; Moravec, P.; Seidl, D. Calibration of Short Range 2D Laser Range Finder for 3D SLAM Usage. J. Sens. 2016, 2016. [CrossRef]

11. Oberlaender, J.; Pfotzer, L.; Roennau, A.; Dillmann, R. Fast Calibration of Rotating and Swivelling 3-D Laser Scanners Exploiting Measurement Redundancies. In Proceedings of the 2015 IEEE/RSJ International Conference on Intelligent Robots and Systems, Hamburg, Germany, 28 September-2 October 2015; pp. 3038-3044.

12. Kurnianggoro, L.; Hoang, V.-D.; Jo, K.-H. Calibration of Rotating 2D Laser Range Finder Using Circular Path on Plane Constraints. In New Trends in Computational Collective Intelligence; Camacho, D., Kim, S.W., Trawinski, B., Eds.; Springer: Cham, Switzerland, 2015; Volume 572, pp. 155-163.

13. Kurnianggoro, L.; Hoang, V.-D.; Jo, K.-H. Calibration of a 2D Laser Scanner System and Rotating Platform using a Point-Plane Constraint. Comput. Sci. Inf. Syst. 2015, 12, 307-322. [CrossRef]

14. Pfotzer, L.; Oberlaender, J.; Roennau, A.; Dillmann, R. Development and calibration of KaRoLa, a compact, high-resolution 3D laser scanner. In Proceedings of the IEEE International Symposium on Safety, Hokkaido, Japan, 27-30 October 2014.

15. Lin, C.C.; Liao, Y.D.; Luo, W.J. Calibration method for extending single-layer LIDAR to multi-layer LIDAR. In Proceedings of the 2013 IEEE/SICE International Symposium on System Integration (SII), Kobe, Japan, 15-17 December 2013.

16. Ueda, T.K.H.; Tomizawa, T. Mobile SOKUIKI Sensor System-Accurate Range Data Mapping System with Sensor Motion. In Proceedings of the 2006 International Conference on Autonomous Robots and Agents, Palmerston North, New Zealand, 12-14 December 2006.

17. Nagatani, K.; Tokunaga, N.; Okada, Y.; Yoshida, K. Continuous Acquisition of Three-Dimensional Environment Information for Tracked Vehicles on Uneven Terrain. In Proceedings of the IEEE International Workshop on Safety, Sendai, Japan, 21-24 October 2008.

18. Matsumoto, M.; Yuta, S. 3D laser range sensor module with roundly swinging mechanism for fast and wide view range image. In Proceedings of the Multisensor Fusion \& Integration for Intelligent Systems, Salt Lake City, UT, USA, 5-7 September 2010.

19. Walther, M.; Steinhaus, P.; Dillmann, R. A foveal 3D laser scanner integrating texture into range data. In Proceedings of the International Conference on Intelligent Autonomous Systems 9-ias, Tokyo, Japan, 7-9 March 2006.

20. Raymond, S.; Nawid, J.; Waleed, K.; Claude, S. A Low-Cost, Compact, Lightweight 3D Range Sensor. In Proceedings of the Australasian Conference on Robotics and Automation; Available online: https://www.researchgate.net/publication/228338590_A_ Low-Cost_Compact_Lightweight_3D_Range_Sensor (accessed on 6 January 2021).

21. Dias, P.; Matos, M.; Santos, V. 3D Reconstruction of Real World Scenes Using a Low-Cost 3D Range Scanner. Comput.-Aided Civ. Infrastruct. Eng. 2006, 21, 486-497. [CrossRef]

22. Nasrollahi, M.; Bolourian, N.; Zhu, Z.; Hammad, A. Designing LiDAR-equipped UAV Platform for Structural Inspection. In Proceedings of 34th International Symposium on Automation and Robotics in Construction; IAARC Publications, 2018. Available online: https: / / www.researchgate.net/publication/328370814_Designing_LiDAR-equipped_UAV_Platform_for_Structural_ Inspection (accessed on 6 January 2021).

23. Bertussi, S. Spin_Hokuyo-ROS Wiki. Available online: http://wiki.ros.org/spin_hokuyo (accessed on 13 October 2020).

24. Bosse, M.C.; Zlot, R.M. Continuous 3D scan-matching with a spinning 2D laser. In Proceedings of the IEEE International Conference on Robotics \& Automation, Kobe, Japan, 12-17 May 2009.

25. Zheng, F.; Shibo, Z.; Shiguang, W.; Yu, Z. A Real-Time 3D Perception and Reconstruction System Based on a 2D Laser Scanner. J. Sens. 2018, 2018, 1-14. [CrossRef]

26. Almqvist, H.; Magnusson, M.; Lilienthal, A.J. Improving Point Cloud Accuracy Obtained from a Moving Platform for Consistent Pile Attack Pose Estimation. J. Intell. Robot. Syst. 2014, 75, 101-128. [CrossRef]

27. Zhang, J.; Singh, S. LOAM: Lidar Odometry and Mapping in Real-time. In Robotics: Science and Systems; 2014. Available online: https:/ /www.researchgate.net/publication/311570125_LOAM_Lidar_Odometry_and_Mapping_in_real-time (accessed on 6 January 2021).

28. Zhang, T.; Nakamura, Y. Moving Humans Removal for Dynamic Environment Reconstruction from Slow-Scanning LIDAR Data. In Proceedings of the 2018 15th International Conference on Ubiquitous Robots, Honolulu, HI, USA, 26-30 June 2018 ; pp. 449-454.

29. David, Y.; Kent, W. “Sweep Diy 3d Scanner Kit” Project. Available online: https://www.servomagazine.com/magazine/article/ the-multi-rotor-hobbyist-scanse-sweep-3d-scanner-review? (accessed on 16 October 2020).

30. Hokuyo UST-10/20LX. Available online: https:/ / www.hokuyo-aut.co.jp/search/single.php?serial=16 (accessed on 16 October 2020). 
31. Point Cloud Library. Available online: https://pointclouds.org/ (accessed on 16 October 2020).

32. More, J.J. The Levenberg-Marquardt algorithm: Implementation and theory. In Lecture Notes in Mathematicsl; Springer: Berlin/Heidelberg, Germany, 1978; Volume 630.

33. Madsen, K.; Nielsen, H.B.; Tingleff, O. Methods for Non-Linear Least Squares Problems, 2nd ed.; Informatics and Mathematical Modelling (IMM), Technical University of Denmark (DTU): Lyngby, Denmark, 2004.

34. Ricaud, B.; Joly, C.; de La Fortelle, A. Nonurban Driver Assistance with 2D Tilting Laser Reconstruction. J. Surv. Eng. 2017, 143. [CrossRef]

35. Yan, F.; Zhang, S.; Zhuang, Y.; Tan, G. Automated indoor scene reconstruction with mobile robots based on 3D laser data and monocular visual odometry. In Proceedings of the 2015 IEEE International Conference on CYBER Technology in Automation, Control, and Intelligent Systems (CYBER), Shenyang, China, 8-12 June 2015.

36. Colas, F.; Mahesh, S.; Pomerleau, F.; Liu, M.; Siegwart, R. 3D path planning and execution for search and rescue ground robots. In Proceedings of the IEEE/RSJ International Conference on Intelligent Robots \& Systems, Tokyo, Japan, 3-7 November 2013. 